\title{
Voting in small networks with cross-pressure*
}

\author{
Ascensión Andina-Díaz ${ }^{\dagger} \quad$ Miguel A. Meléndez-Jiménez ${ }^{\ddagger}$
}

November 2006

\begin{abstract}
We present a model of participation in elections in small networks, in which citizens suffer from cross-pressures if voting against the alternative preferred by some of their social contacts. We analyze how the existence of cross-pressures may shape voting decisions, and so, political outcomes; and how candidates may exploit this effect to their interest.
\end{abstract}

JEL classification: D72

Keywords: Network; Voting; Cross-Cutting.

*The authors thank Steven Callander, Daniel Diermeier, Jeff Ely and Stanley Reiter for their comments. Ascensión Andina-Díaz also thanks the hospitality of Kellogg School of Management, Northwestern University, while working on this paper. The usual disclaimer applies.

${ }^{\dagger}$ Departamento de Fundamentos del Análisis Económico, Universidad de Murcia, 30100 Espinardo Murcia, Spain. E-mail: asandina@um.es

${ }^{\ddagger}$ CMS-EMS, Kellogg School of Management, Northwestern University, Evanston, IL 60208, USA; and Departamento de Teoría e Historia Económica, Universidad de Málaga, 29071 Málaga, Spain. E-mail: m-melendez@northwestern.edu 
"To go against the dominant thinking of your friends, of most of the people you see every day, is perhaps the most difficult act of heroism you can perform".

Theodore H. White

\section{Introduction}

Scholars devoted to the study of voting behavior have placed much of their attention on the reasons explaining the decision to cast a vote. Their interest on this issue comes from the necessity to understand the "paradox of not voting", namely, why voters, in large elections, engage in costly voting if their probability of being pivotal, and therefore their probability of changing the outcome of an election, is vanishingly small. It has been argued and posteriori shown by empirical literature, that turnout levels are inversely related to the cost of voting (Wolfinger and Rosenstone (1980), or Riker and Ordeshook (1968) among others). The classic factors usually considered in the literature to impact the costs of voting are the weather, the distance to the pooling place, or the information and the time required to think about one's voting decision. Little if no attention has been placed, however, on the existence of cross-pressures that may undermine the incentives and willingness to vote.

Nevertheless, empirical research on the US has found that people experiencing conflicts and inconsistencies between their opinions and those of their relatives, friends or co-workers, are less likely to participate in politics. Mutz (2002), in an empirical study for the US presidential and congressional elections of the 1992 and 1996, finds that the probability of voting in an election is positively related to some of the usual predictors such as political interest, partisanship, education or age. But she also finds that the decision to cast a vote is strongly negatively related to the exposition to dissonant political opinions within one's personal network. Mutz (2002) documents two theories to explain this effect. First, that cross-cutting exposure is likely to engender attitudinal ambivalence to an individual, inducing political inaction. Second, that cross-pressures arising from one's personal network can create the need to be socially accountable, and then, it may bring about uncomfortable feelings when facing a decision that does not please everybody in one's network. As a result, individuals embedded in networks that supply them with political information that challenges their views can be discouraged from involving and participating in politics.

Apart from Mutz (2002), there is a number of studies pointing out to the problems posed by the existence of cross-pressures, such as Lazarsfeld et al. (1944), Simmel (1955), Campbell et al. (1960), Hibbing and Theiss-Morse (2002) or Mutz (2006). All these studies are empirical and they all base on the analysis of data from social and political surveys. There is not, however, and to the best of our knowledge, any theoretical study in which this phenomenon has been formally introduced. This is the aim of this paper, in which we want to analyze how the existence of cross-pressures may shape voting 
decisions, and so, political outcomes; and how political parties may exploit this effect to their interest.

To this aim, we consider a winner-take-all election game with two candidates (an incumbent and a challenger) representing two different alternatives or ideologies. We consider a finite number of citizens that belong to one of the two groups or factions depending on their own preferred alternative. There is a network representing the structure of friendship or close relationship among the citizens in the community, i.e., a link between two agents represents the idea that they are close friends and not merely acquaintances. We focus our attention on the study of small communities or small networks, where the pattern of friendship relationships within the community and the preferences of all the individuals are known to everybody, and so the existence of cross-pressures becomes of a special relevance. We consider that candidates nominate sequentially (first the challenger and then the incumbent) a certain number of citizens, within those rooting for their same preferred alternative, to run for office in support of that alternative. This idea of small networks and a candidate making lists of citizens to be elected, may apply to a number of situations. For example, in the field of the academics, we can think of elections for the board of directors in a department (chair, vice-chair and secretary), or elections for the board of directors in a college (dean and associate deans). Similarly, the elections for the board of directors in private firms or soccer clubs work, in some cases, this way. Additionally, it can also apply to elections for mayor in villages or small towns in many European countries, like Austria, Finland, Italy, Spain or Turkey, where parties make lists of nominees to be elected.

We introduce the effect of the cross-cutting cost in the model in a very stylized way, which, however, allows us to obtain some sharp results. We assume that an individual suffers from cross-pressure when he has at least one friend with a cross-preferred alternative running for office, and additionally, he votes for his own preferred alternative (therefore against his friend). As already pointed out, empirical evidence documenting the effects of cross-pressures finds the existence of political disagreement sufficient to generate this cost. We are, however, much more restrictive and consider the existence of cross-pressure only when, with your vote, you "hurt" some of your friends. By so doing, we clearly define the crosscutting cost, although it implies it to be considered a much more limited phenomenon.

The existence of cross-pressures will lead certain citizens in our model to abstain from voting, in particular, those for whom the benefit of having their preferred alternative implemented does not pay the cost of bearing the cross-cutting effect. This, additionally, will lead the candidates to strategically choose their nominees so as to exploit this effect.

Because of two types of coordination problems among citizens that may arise in this model, our equilibrium concept is the strong perfect equilibrium. The strong Nash condition requires an equilibrium to be immune to every possible coalitional deviation, and as usual, the perfect condition says that a strategy profile must remain an equilibrium strategy profile in every proper subgame of the game.

This stylized model delivers some sharp results. Our first result is that there always exists a strong 
perfect equilibrium. Our second result says that, even though there may exist more than one strong perfect equilibrium for our game, they all are outcome equivalent in terms of which is the alternative winning office. This result is important for our analysis as it allows us to restrict our attention to a subset of strong perfect equilibria that satisfies some reasonable conditions, as it is that players use weakly dominant strategies, and that candidates maximize (minimize) the difference of votes if this difference is favorable (unfavorable) to them. It is important to note that these conditions, although restrict and simplify the model, do not have any consequence in terms of the outcome, namely they do not alter which is the alternative getting into power. Our third result refers to the candidates' optimal strategies, and it establishes the conditions that the optimal strategy of a candidate must satisfy. From this result we derive one implication for the case in which the candidates make lists of just one citizen, as it is that, in such a case, the candidates nominate in equilibrium the citizen (or one of the citizens) with more friends in the other group. That is to say, candidates find it optimal to nominate the citizen that "covers" (have links to) the largest number of people in the other faction, and so, generates the highest amount of pressure in the other side. This equilibrium displays the feature known in graph theory as maximal covering (see, for instance, Asratian et al.(1998)). This quite intuitive result fails to hold, however, in the case each candidate has to nominate more than one citizen. It turns out that, in such a case, a candidate's optimal strategy has to take into account not only the covering of a list, but also the difference between the citizens running for office covered by the two lists. Hence, we show by a counter-example that, in this case, there are not necessarily equilibria in maximal covering, as it may be the case that a list that is not a maximal covering procures a favorable difference of covered running-citizens that outweighs its smaller covering. We next derive some results on the importance of being the last mover. Here we obtain that, in the case each candidate proposes a list of just one citizen, the identity of the candidate getting into power does not depend on who is the incumbent at the beginning of the game, but it is simply determined by the covering of the two lists. This result, however, is not generally true when the lists are of two of more citizens each. In such a case, the incumbent has generally the advantage of being the last mover of the game. Finally, we analyze the equilibria of the game for extreme values of the cross-cutting cost (instead of more intermediate values of this cost as it is assumed in the main body of the paper). We observe that, for low values of the cross-cutting cost, an equilibrium may generally fail to exist. On the other hand, for high values of cross-pressures we observe that the existence of such high costs favors the first mover, i.e., challenger, as compared to the case of an intermediate cost.

The idea of this paper emerged after reading the work by Mutz (2002). Mutz (2002) crystallizes insights from earlier theories within social psychologists and political sociologists, pointing out to the negative effects posed by cross-pressures on voting behavior. This is quite a long-standing debate within these disciplines, with some classical studies such as Lazarsfeld et al., (1944), Simmel (1955) or Campbell et al. (1960). However, none of these classical studies were successful in confirming that people involved 
in cross-cutting networks were induced either political ambivalence or a need to be socially accountable that finally discouraged them from participating in politics. Mutz (2002), differently, takes advantage of the more complete data set available nowadays and uses two surveys, the Cross-National Election Project (CNEP), executed during the 1992 US election, and the Spencer survey, carried out during the fall of 1996, just before the US presidential election in November; to show that "People whose network involve greater political disagreement are less likely to participate (vote) in politics".

Our model fits into the literature on voter turnout, pioneered by Riker and Ordeshook (1968), Ledyard (1981, 1984) and Palfrey and Rosenthal $(1983,1985)$ among others. More recent contributions to this literature embedded into the "group-based" models of turnout, so called because they coincide on the idea that the act of voting needs to be understood as a group decision. This "group-based" models, such as Uhlaner (1989), Morton (1991), Shachar and Nalebuff (1999), or Herrera and Martinelli (2006), consider that voters participate in elections because they are mobilized by leaders, who happen to affect the voting decisions of affine citizens by means of side-payments or social pressure (see Feddersen (2004) for a recent survey). Our model is close to this literature in that our leaders (candidates and running-citizens) also compete for political support, although in our case they do it by means of demobilizing, rather than mobilizing, the electorate. We differ from theirs in that we endogenize the choice of leaders (nominees), and in the key feature that we consider a network structure, with all its richness and particularities, that imposes heterogeneity among all our agents.

In the same vein of mobilization models, the literature on vote buying considers that vote buyers make offers to purchase votes so as to favor their preferred policy. Groseclose and Snyder (1996) considers a model of sequential vote buying in a legislature, where the two-period game form provides the last mover an important advantage. This second mover advantage does also appear in our game. Recently, Dekel et al. (2006a,b) analyze a sequential vote buying game where the number of "bribing" rounds is determined endogenously, and the structure of the game does not therefore provide an advantage to any of the parties.

Within the literature on social networks, Fowler (2005) is probably the paper closest to ours, who studies, for a "small world" network, how an individual's voting decision spreads within the network. Fowler attempts to explain the striking turnout numbers in large elections, and he provides an answer based on the idea that if voters imitate each other's behavior, then a single voting decision may generate a turnout cascade.

In the analysis that follows, we present the model and the structure of the game in Section 2. In Section 3, we formally define the equilibrium notion and characterize the equilibrium conditions of the game. In Section 4, we make a deeper analysis of the equilibrium behavior of candidates. In Section 5, we relax some of our assumptions and study the equilibria of the game for both high and low values of the cross-cutting cost. Finally we conclude in Section 6. 


\section{The model}

We analyze a winner-take-all election with two candidates $A$ and $B$ representing alternatives or ideologies $\mathcal{A}$ and $\mathcal{B}$ respectively. There is a finite population of citizens $N$. We denote by $N_{\mathcal{A}}$ the set of citizens favoring alternative $\mathcal{A}$, and by $N_{\mathcal{B}}$ the set of citizens favoring alternative $\mathcal{B}$, with $N_{\mathcal{A}} \cup N_{\mathcal{B}}=N$ and $N_{\mathcal{A}} \cap N_{\mathcal{B}}=\emptyset$. For simplicity, we assume that neither $A$ nor $B$ belong to $N$.

There is an undirected network $g: N \times N \rightarrow\{0,1\}$ representing the structure of friendship among the elements of $N$, where $g(x, y)=1$ if there is a link between citizens $x$ and $y$, i.e., citizens $x$ and $y$ are friends or have a close relationship, and $g(x, y)=0$ otherwise. ${ }^{1}$ Let us define, for any $i \in N_{\mathcal{A}}$, $F(i)=\left\{j \in N_{\mathcal{B}}: g(i, j)=1\right\}$ as the set of friends of citizen $i$ that favor ideology $\mathcal{B}$. Analogously, for any $j \in N_{\mathcal{B}}, F(j)=\left\{i \in N_{\mathcal{A}}: g(i, j)=1\right\}$ represents the set of friends of citizen $j$ that favor ideology $\mathcal{A}$.

We analyze the full information version of the game. That is to say, we consider that the network structure and the preferences of all the agents are known to everybody. This is not an extreme assumption in this model, as we want to analyze voting in small networks or small communities, where everybody knows each other and knows each other's preferences. This does not imply, however, that there is a close relationship among all the agents in the community, but the structure of friendships is determined by the network $g$.

We consider elections in which there are $k+1$ seats in office for the winning alternative, with $k \in$ $\mathbb{N} \backslash\{0\} .{ }^{2}$ We assume that $k \leq \min \left\{\left|N_{\mathcal{A}}\right|,\left|N_{\mathcal{B}}\right|\right\}$. At the beginning of the game we consider that there is one candidate in office, without loss of generality, candidate $A$. That is to say, at the beginning of the game, candidate $A$ and a list $\widehat{l}_{A}$ of $k$ citizens in $N_{\mathcal{A}}$, representing alternative $\mathcal{A}$, are in power. We consider a three stage game with timing as follows:

First stage (challenging stage): The challenger, candidate $B$, chooses which $k$ citizens, from the set $N_{\mathcal{B}}$, nominate to join her and comprise the list that will challenge the incumbent, i.e., she chooses a list from the set $L_{B}=\left\{l_{B} \subseteq N_{\mathcal{B}}:\left|l_{B}\right|=k\right\}$. The nominees are informed about the identity of the $k$ proposed citizens and then each nominee decides, simultaneously, whether to accept or not to be part of that list. In the case at least one of the proposed citizens does not accept, the game finishes: no challenging list forms, and the incumbent keeps in office. The reader can think of this situation as a non contested election in which the only alternative available is the one currently being implemented. For simplicity we assume that, in such a case, there is no election and the incumbent remains in office. ${ }^{3}$

\footnotetext{
${ }^{1}$ The fact that $g$ is undirected means that $g(x, y)=g(y, x) \forall x, y \in N$.

${ }^{2}$ For example, elections for board of directors or governance committees in university departments or firms.

${ }^{3}$ However, the result would not change if we considered that an election takes place in such a case. The reason is that the citizens currently at office always want to run for a new election if they know that they will win for sure, as it is here the case. This means that the incumbent proposes the same list, and the citizens in favor of this alternative vote for it (this is, at least, a weakly dominant strategy for the citizens). As a result, the incumbent, with or without elections, keeps in office if the challenger is not able to form a list.
} 
Differently, if all of the proposed citizens accept, the list representing alternative $\mathcal{B}$ is formed, and we move to the second stage.

Second stage (response stage): At the beginning of the second stage all the citizens in $N$ and the two candidates observe the challenging list. The incumbent, candidate $A$, chooses which $k$ citizens, from the set $N_{\mathcal{A}}$, nominate to join her and comprise the new list that will contest the challenger, i.e., she chooses a list from the set $L_{A}=\left\{l_{A} \subseteq N_{\mathcal{A}}:\left|l_{A}\right|=k\right\} .{ }^{4}$ The nominees are informed about the identity of the $k$ proposed citizens and then each nominee decides, simultaneously, whether to accept or not to be part of that list. Again, if at least one of the proposed citizens does not accept, the game finishes: the incumbent is not able to form a list that will threaten the challenger and then the challenger gets into office. ${ }^{5}$ Differently, if all of the proposed citizens accept, the new list representing alternative $\mathcal{A}$ is formed, and we move to the third stage of the game.

Third stage (voting stage): Each citizen $x \in N$ decides whether to vote for the alternative he favors or to abstain. We assume that the candidates, $A$ and $B$, always vote for their preferred alternative. The alternative with more votes gets into office. In case of a tie, a coin flip determines the winner.

We next define the strategies for candidates and citizens. We focus on pure strategies. A strategy for candidate $B, s_{B}$, is an element from the set $S_{B}=L_{B}$, i.e., a strategy for candidate $B$ is to nominate the $k$ citizens that will represent alternative $\mathcal{B}$. A strategy for candidate $A, s_{A}$, is a function $s_{A}: L_{B} \rightarrow L_{A}$ that maps any possible list $l_{B} \in L_{B}$ formed in the first stage, into a list $l_{A} \in L_{A}$ of $k$ citizens, representing alternative $\mathcal{A}$, to contest $l_{B}$. Let $S_{A}$ be the set of strategies of candidate $A$.

Regarding citizens, they have two choices to make. They first have to decide whether to accept or not to run for office, if proposed to be part of a list; and then have to decide whether to vote or not for their most preferred alternative. A strategy for citizen $j \in N_{\mathcal{B}}$ is then $\left(r_{j}, v_{j}\right)$, where $r_{j}: L_{B} \rightarrow\{0,1\}$ is a function that represents the decision of whether to accept to run for office, $r_{j}\left(l_{B}\right)=1$, or not to accept it, $r_{j}\left(l_{B}\right)=0$, if proposed by candidate $B$ in the first stage, given list $l_{B}{ }^{6}$ and $v_{j}: L_{A} \times L_{B} \rightarrow\{0,1\}$ is a function that represents the decision of whether to vote for alternative $\mathcal{B}, v_{j}\left(l_{A}, l_{B}\right)=1$, or to abstain, $v_{j}\left(l_{A}, l_{B}\right)=0$, in the third stage, given the two lists. Analogously, a strategy for citizen $i \in N_{\mathcal{A}}$ is $\left(r_{i}, v_{i}\right)$, where $r_{i}: L_{A} \times L_{B} \rightarrow\{0,1\}$ is a function that represents the decision of whether to accept to run for office, $r_{i}\left(l_{A}, l_{B}\right)=1$, or not, $r_{i}\left(l_{A}, l_{B}\right)=0$, if proposed by candidate $A$ in the second stage, given lists $l_{A}$ and $l_{B} ;^{7}$ and $v_{i}: L_{A} \times L_{B} \rightarrow\{0,1\}$ is a function that represents the decision of whether to vote for

\footnotetext{
${ }^{4}$ Note that we do not impose that candidate $A$ contests the challenger with the list of citizens $\widehat{l}_{A}$ initially in power. We do allow her, however, to choose a new list afresh.

${ }^{5}$ Here again we assume, for simplicity, that no election is held if it is not contested. The reader may note, however, that an election would yield the same result, i.e., the unique list running for office (candidate $B$ 's list) gets into power.

${ }^{6}$ Note that, given a proposed list $l_{B} \in L_{B}$, an individual $j \in N_{\mathcal{B}}$ has to decide whether to accept to be part of that list only if $j \in l_{B}$. However, in order to define a strategy profile in a compact way, we consider that each agent $j \in N_{\mathcal{B}}$ chooses $r_{j}\left(l_{B}\right) \in\{0,1\}$ for all $l_{B} \in L_{B}$, regardless of whether $j \in l_{B}$ or not. Clearly, the choice of all the agents in the set $N_{\mathcal{B}} \backslash l_{B}$ is irrelevant.

${ }^{7} \mathrm{By}$ a similar argument to the one used in the previous footnote, we also consider that each agent $i \in N_{\mathcal{A}}$ chooses
} 
alternative $\mathcal{A}, v_{i}\left(l_{A}, l_{B}\right)=1$, or to abstain, $v_{i}\left(l_{A}, l_{B}\right)=0$, in the third stage, given the two lists. Let $S_{i}$ and $S_{j}$ denote the set of strategies to any citizen $i \in N_{\mathcal{A}}$ and $j \in N_{\mathcal{B}}$, respectively.

Let $S$ be the set of (pure) strategy profiles of the game, with

$$
s=\left(s_{A}\left(l_{B}\right), s_{B},\left\{\left(r_{i}\left(l_{A}, l_{B}\right), v_{i}\left(l_{A}, l_{B}\right)\right)\right\}_{i \in N_{\mathcal{A}}},\left\{\left(r_{j}\left(l_{B}\right), v_{j}\left(l_{A}, l_{B}\right)\right)\right\}_{j \in N_{\mathcal{B}}}\right) \in S .
$$

We define a function $R_{B}: S \rightarrow\{0,1\}$ representing the idea of whether candidate $B$, supporting alternative $\mathcal{B}$, has successfully formed a list and is then running for office. In particular, $R_{B}(s)=$ $\min _{j \in s_{B}}\left\{r_{j}\left(s_{B}\right)\right\}$, where $R_{B}(s)=1$ if all the nominees accept to be in the list $s_{B}$ and, therefore, candidate $B$ (with list $s_{B}$ ) runs for office; and $R_{B}(s)=0$ if at least one of the nominees does not accept and, hence, the list is not formed. Analogously, we define the function $R_{A}: S \rightarrow\{0,1\}$, as $R_{A}(s)=\min _{i \in s_{A}\left(s_{B}\right)}\left\{r_{i}\left(s_{A}\left(s_{B}\right), s_{B}\right)\right\}$.

The number of votes for alternative $\mathcal{A}$ is $1+\sum_{i \in N_{\mathcal{A}}} v_{i}\left(s_{A}\left(s_{B}\right), s_{B}\right)$, i.e., the vote of candidate $A$ and those of citizens in $N_{A}$ that cast their vote. Analogously, the number of votes for alternative $\mathcal{B}$ is $1+\sum_{j \in N_{\mathcal{B}}} v_{j}\left(s_{A}\left(s_{B}\right), s_{B}\right)$. We consider a winner-take-all election, and so, the alternative with more votes gets into office. In case of a tie, a coin flip determines the winner. Hence, given $s \in S$, the probability that alternative $\mathcal{A}$ gets into office is

$P_{A}(s)= \begin{cases}1 & \text { if either } R_{B}(s)=R_{A}(s)=1 \text { and } \sum_{i \in N_{\mathcal{A}}} v_{i}\left(s_{A}\left(s_{B}\right), s_{B}\right)>\sum_{j \in N_{\mathcal{B}}} v_{j}\left(s_{A}\left(s_{B}\right), s_{B}\right), \text { or } R_{B}(s)=0 \\ 1 / 2 & \text { if } R_{B}(s)=R_{A}(s)=1 \text { and } \sum_{i \in N_{\mathcal{A}}} v_{i}\left(s_{A}\left(s_{B}\right), s_{B}\right)=\sum_{j \in N_{\mathcal{B}}} v_{j}\left(s_{A}\left(s_{B}\right), s_{B}\right) \\ 0 & \text { otherwise, }\end{cases}$

and the probability that alternative $\mathcal{B}$ does is $P_{B}(s)=1-P_{A}(s)$.

In this model we want to capture the idea that voting in a small network, where everything is common knowledge and relationships are close, may imply a cost for a citizen if he casts his ballot for other alternative rather than the one preferred in his social network. That is to say, voting for your preferred alternative, but against the option preferred by your friends, can create cross-pressures that citizens may want to avoid. In this model we capture this idea in a very stylized way. In particular, we assume that citizen $x$ suffers from cross-cutting pressures if at least one of his contacts (friends or close-colleagues) in the social network runs for office supporting the alternative he does not favor and either citizen $x$ votes (for his preferred alternative) or he also belongs to a list. This means that, unless running for office, to abstain is a way to avoid the cross-cutting cost. Let us denote by $c$ this cross-cutting cost. ${ }^{8}$

We consider that holding office is attractive. More precisely, we assume that candidates and citizens attach a benefit of $h$ if they hold office. Additionally, we consider that candidates and citizens get a benefit of $d$ if their most preferred alternative is implemented.

\footnotetext{
$r_{i}\left(l_{A}, l_{B}\right) \in\{0,1\}$ for all $\left(l_{A}, l_{B}\right) \in L_{A} \times L_{B}$.

${ }^{8}$ Note that candidates $A$ and $B$ do not incur in cross-cutting pressures as we assume that they do not belong to the network and, therefore, do not have friends in the other faction. Nevertheless, our results would not change if we considered that candidates belong to the network but only have friends among those ideologically affine to theirs.
} 
We can now specify the payoffs of citizens in $N$ and those of candidates. Given $s \in S$, the payoff of an agent $i \in N_{\mathcal{A}} \cup\{A\}$ is

$$
\pi_{i}(s)=P_{A}(s)\left(h_{i}(s)+d\right)-c_{i}(s)
$$

where:

- $h_{i}(s)$ is a function representing the benefit of holding office, with $h_{i}(s)=h$ if either agent $i$ is running for office $\left(i \in s_{A}\left(s_{B}\right) \cup\{A\}\right.$ and $R_{B}(s)=R_{A}(s)=1$ ) or he is already at office and the challenger does not threaten the incumbent $\left(i \in \hat{l}_{A} \cup\{A\}\right.$ and $\left.R_{B}(s)=0\right)$; and $h_{i}(s)=0$ otherwise,

- $d$ is the benefit of having his preferred ideology $\mathcal{A}$ implemented, and

- $c_{i}(s)$ is a function representing the cross-cutting cost, with $c_{i}(s)=c$ if agent $i$ has at least one friend running for alternative $\mathcal{B}\left(R_{B}(s)=R_{A}(s)=1\right.$ and $\left.F(i) \cap s_{B} \neq \emptyset\right)$ and, either agent $i$ runs for alternative $\mathcal{A}\left(i \in s_{A}\left(s_{B}\right)\right)$ or he votes for alternative $\mathcal{A}\left(v_{i}\left(s_{A}\left(s_{B}\right), s_{B}\right)=1\right)$; and $c_{i}(s)=0$ otherwise. Note that candidate $A$ does not incur in the cross-cutting cost, i.e., $c_{A}(s)=0$ for all $s \in S$ (cf. footnote 8).

Analogously, the payoff of an agent $j \in N_{\mathcal{B}} \cup\{B\}$ is $\pi_{j}(s)=P_{B}(s)\left(h_{j}(s)+d\right)-c_{j}(s)$, where $h_{j}(s)$ is an analogous function representing the benefit of holding office, $d$ is the benefit of having his preferred ideology $\mathcal{B}$ implemented, and $c_{j}(s)$ is an analogous function representing the cross-cutting cost.

Note that the existence of the cross-cutting cost affects not only the decision of whether to vote or not, but it also affects the decision of whether to accept to be part of a list. This is so because we assume that agents in a list incur in the cross-cutting cost when they have at least one friend running for their cross-preferred alternative, independently on whether they vote or not.

In the analysis that follows that constitutes the main body of the paper, we assume $c>d>0$ and $h>2 c-d$. Nevertheless, these two assumption are relaxed on Section 5 , where we analyze the game for the cases of a low $(c<d)$ and a high $(c>h)$ cross-cutting cost. Regarding the assumptions $c>d>0$ and $h>2 c-d$ that apply in the main body of the paper, we observe: (i) The first condition says that the cross-cutting cost is more important for any citizen that the benefit of having his preferred alternative implemented, i.e., any citizen who is not in a list and has a friend with a cross preferred alternative who is running for office, gets a higher payoff by abstaining than by voting; (ii) The second condition says that holding office is sufficiently attractive, i.e., it is profitable for any nominee who anticipates that his list will win or tie in an election to accept to be part of that list.

\section{Equilibrium analysis}

\subsection{The equilibrium notion}

In the multistage setup proposed in our game it is reasonable to require subgame perfection to the Nash concept. However, as we shall explain below, our model engenders two coordination problems, which 
result in a huge multiplicity of subgame perfect equilibria that are not necessarily outcome equivalent. ${ }^{9}$

The first coordination problem that generates multiplicity of subgame perfect equilibria appears when the nominees have to decide whether to accept or not to be part of a list. For example, if $k \geq 2$ and $r_{j_{1}}\left(s_{B}\right)=r_{j_{2}}\left(s_{B}\right)=0$ for at least two nominees $j_{1}, j_{2} \in s_{B}$, then $R_{B}(s)=\min _{j \in s_{B}}\left\{r_{j}\left(s_{B}\right)\right\}=0$ and therefore any acceptance decision for each of the remaining nominees in $s_{B}$ can be sustained in a subgame perfect Nash equilibrium. In such equilibria, the challenger is not able to form a list and so the incumbent's list keeps in office. Analogously, the nominees in the list $s_{A}\left(s_{B}\right)$ may face the same coordination problem. Thus, given any list $s_{B}$, if $k \geq 2$ and $r_{i_{1}}\left(s_{A}\left(s_{B}\right), s_{B}\right)=r_{i_{2}}\left(s_{A}\left(s_{B}\right), s_{B}\right)=0$ for at least two nominees $i_{1}, i_{2} \in s_{A}\left(s_{B}\right)$, then $R_{A}(s)=0$ and so any acceptance decision for each of the remaining nominees in $s_{A}\left(s_{B}\right)$ can be sustained in a subgame perfect Nash equilibrium. In such equilibria, the incumbent is not able to form a list to contest the challenger, therefore the latter gets into office. This first coordination problem leads to the existence of multiple subgame perfect Nash equilibria, which are not necessarily outcome equivalent, and with the common feature that in all of them a list is not formed, even though it may be profitable for all of the proposed nominees to (jointly) accept to be in the list.

The second coordination problem that generates multiplicity of subgame perfect Nash equilibria arises when the citizens have to decide whether to vote or to abstain. To illustrate this problem, let us consider a strategy leading to an outcome in which there are at least two agents in $N_{\mathcal{B}}$ voting for alternative $\mathcal{B}$, whereas no agent in $N_{\mathcal{A}}$ votes for alternative $\mathcal{A}$. In such a case, those agents in $N_{\mathcal{A}}$ who, for the given pair of lists, do not have cross pressures, are indifferent between voting and not voting. Hence, the multiplicity of equilibria. Note however that, if the number of such agents were higher than the number of citizens voting for alternative $\mathcal{B}$, they would all benefit if they all were to vote.

To address these coordination problems, we use a refinement that allows for coalitional deviations. The two most well-known refinements proposed in the literature meant to capture the possibility of coordinated deviations are, possibly, the strong Nash equilibrium (Aumann (1959)) and the coalition-proof Nash equilibrium (Bernheim et al. (1987)). These two concepts require an equilibrium to be immune to coalitional deviations. The difference between them is, however, that while the strong Nash equilibrium allows for deviations by every possible coalition, the coalition-proof Nash equilibrium requires that deviations must be self-enforcing, i.e., no proper sub-coalition can beneficially deviate from the deviation. Thus, the strong Nash equilibrium is more restrictive than the coalition-proof Nash equilibrium. In fact, the coalition-proof Nash equilibrium was defined in view of the existence problems that the strong Nash equilibrium was shown to present in many games. The game we analyze is, however, such that there always exists a strong Nash equilibrium, or more precisely, a strong perfect equilibrium, which is the precise concept that we use. ${ }^{10}$ This concept adapts the strong Nash equilibrium notion to the class of

\footnotetext{
${ }^{9}$ Note that the concept of subgame perfect equilibria is based on the implicit assumption that players cannot coordinate on any joint, mutually beneficial, change of strategies.

${ }^{10}$ This is true given the conditions $c>d>0$ and $h>2 c-d$, which we assume throughout the main body of the paper.
} 
sequential-move games, by simply imposing a strategy profile to be a strong Nash equilibrium in every proper subgame of the game.

We now formally define the equilibrium notion. For each subset $C \subseteq N \cup\{A\} \cup\{B\}$, we define $S_{C}=\prod_{z \in C} S_{z}$ and $S_{-C}=\prod_{z \notin C} S_{z}$. We first define the notion of strong Nash equilibrium, which was introduced by Aumann (1959).

Definition 1 A strong Nash equilibrium is a strategy profile $s^{*} \in S$ such that for each coalition $C$ and strategy $\hat{s}_{C} \in S_{C}$ there exists at least one agent $z \in C$ such that $\pi_{z}\left(\hat{s}_{C}, s_{-C}^{*}\right) \leq \pi_{z}\left(s^{*}\right)$.

We now add the requirement of subgame perfection to the equilibrium concept and define the notion of strong perfect equilibrium, which was first introduced by Rubinstein (1979) for repeated games with infinite horizon, and posteriorly generalized by Brusco (1996).

Definition 2 A strong perfect equilibrium is a strategy profile $s^{*} \in S$ which is a strong Nash equilibrium for each proper subgame of the extensive form game. Let $S^{*} \subset S$ be the set of strong perfect equilibria.

\subsection{Equilibrium characterization}

We first introduce the conditions that characterize the set of strong perfect equilibria of the game. To this aim, let us define some sets that will play a crucial role in the characterization.

Definition 3 Given a pair of lists $\left(l_{A}, l_{B}\right) \in L_{A} \times L_{B}$, let $I\left(l_{A}, l_{B}\right)=l_{A} \cup\left\{i \in N_{A}: l_{B} \cap F(i)=\emptyset\right\}$ and $J\left(l_{A}, l_{B}\right)=l_{B} \cup\left\{j \in N_{B}: l_{A} \cap F(j)=\emptyset\right\}$.

In words, the set $I\left(l_{A}, l_{B}\right)$ encompasses those individuals favoring alternative $\mathcal{A}$ that, given two lists $l_{A}$ and $l_{B}$ formed and running for office, either incur or do not incur in the cross-cutting cost $c$ regardless of their decision in the voting stage, i.e., those individuals for whom the voting decision does not imply a cost. This set is clearly composed by the members of the list $l_{A}$ (who incur in the cross-cutting cost if they have at least one friend in $l_{B}$ and do not incur in the cost otherwise) and by those citizens in $N_{\mathcal{A}}$ who do not have friends in $l_{B}$, and therefore, do not suffer from cross-pressures. Analogously, the set $J\left(l_{A}, l_{B}\right)$ encompasses those individuals favoring alternative $\mathcal{B}$ that either incur or do not incur in the cross-cutting cost $c$ regardless of their decision in the voting stage. As we shall see below, these sets are crucial to determine the outcome of an election, given a pair of lists $l_{A}$ and $l_{B}$ already formed.

Definition 4 Given a list $l_{B} \in L_{B}$, let $W_{A}\left(l_{B}\right)=\left\{l_{A} \in L_{A}:\left|I\left(l_{A}, l_{B}\right)\right|>\left|J\left(l_{A}, l_{B}\right)\right|\right\}$ and $T\left(l_{B}\right)=$ $\left\{l_{A} \in L_{A}:\left|I\left(l_{A}, l_{B}\right)\right|=\left|J\left(l_{A}, l_{B}\right)\right|\right\}$. Let $\tilde{W}_{B}=\left\{l_{B} \in L_{B}: W_{A}\left(l_{B}\right) \cup T\left(l_{B}\right)=\emptyset\right\}$ and $\widetilde{T}=\left\{l_{B} \in L_{B}\right.$ : $W_{A}\left(l_{B}\right)=\emptyset$ and $\left.T\left(l_{B}\right) \neq \emptyset\right\}$.

When we relax these conditions a strong perfect equilibrium may fail to exist (see Section 5). 
In words, given any list that candidate $B$ may propose, the set $W_{A}\left(l_{B}\right)$ encompasses those lists for candidate $A$ such that, once proposed and formed, the number of citizens favoring alternative $\mathcal{A}$ whose cross-cutting cost is independent of their voting decision exceeds the respective number of citizens favoring alternative $\mathcal{B}$. Similarly $T\left(l_{B}\right)$ encompasses those lists for candidate $A$ such that those numbers are equal. Finally $\tilde{W}_{B}$ represents the set of lists for candidate $B$ for which both $W_{A}\left(l_{B}\right)$ and $T\left(l_{B}\right)$ are empty, and $\tilde{T}$ represents the set of lists for candidate $B$ such that $W_{A}\left(l_{B}\right)$ is empty but $T\left(l_{B}\right)$ is not. As we shall see below, the sets $\tilde{W}_{B}$ and $\tilde{T}$ are crucial to determine the equilibrium outcome of the game (see Proposition 1). With this notation at hand, in the next lemma we characterize the set of strong perfect equilibria of the game.

Lemma 1 A strategy profile $s^{*}=\left(s_{A}^{*}\left(l_{B}\right), s_{B}^{*},\left\{\left(r_{i}^{*}\left(l_{A}, l_{B}\right), v_{i}^{*}\left(l_{A}, l_{B}\right)\right)\right\}_{i \in N_{\mathcal{A}}},\left\{\left(r_{j}^{*}\left(l_{B}\right), v_{j}^{*}\left(l_{A}, l_{B}\right)\right)\right\}_{j \in N_{\mathcal{B}}}\right) \in$ $S$ is a strong perfect equilibrium of the game if and only if the following conditions (1)-(6) hold.

(1) For any $\left(l_{A}, l_{B}\right) \in L_{A} \times L_{B}, v_{z}^{*}\left(l_{A}, l_{B}\right)=0$, for any $z \in N \backslash\left(I\left(l_{A}, l_{B}\right) \cup J\left(l_{A}, l_{B}\right)\right)$.

(2) For any $\left(l_{A}, l_{B}\right) \in L_{A} \times L_{B}:\left(2\right.$ a) if $\left|I\left(l_{A}, l_{B}\right)\right|>\left|J\left(l_{A}, l_{B}\right)\right|$ then $\sum_{i \in N_{\mathcal{A}}} v_{i}^{*}\left(l_{A}, l_{B}\right)>\left|J\left(l_{A}, l_{B}\right)\right|$; (2.b) if $\left|I\left(l_{A}, l_{B}\right)\right|=\left|J\left(l_{A}, l_{B}\right)\right|$ then $\sum_{i \in N_{\mathcal{A}}} v_{i}^{*}\left(l_{A}, l_{B}\right)=\sum_{j \in N_{\mathcal{B}}} v_{j}^{*}\left(l_{A}, l_{B}\right)=\left|I\left(l_{A}, l_{B}\right)\right|$; and (2.c) if $\left|I\left(l_{A}, l_{B}\right)\right|<\left|J\left(l_{A}, l_{B}\right)\right|$ then $\sum_{j \in N_{\mathcal{B}}} v_{j}^{*}\left(l_{A}, l_{B}\right)>\left|I\left(l_{A}, l_{B}\right)\right|$.

(3) For any $\left(l_{A}, l_{B}\right) \in L_{A} \times L_{B}:$ (3.a) if $\left|I\left(l_{A}, l_{B}\right)\right| \geq\left|J\left(l_{A}, l_{B}\right)\right|$ then $r_{i}^{*}\left(l_{A}, l_{B}\right)=1$, for any $i \in l_{A}$; and (3.b) if $\left|I\left(l_{A}, l_{B}\right)\right|<\left|J\left(l_{A}, l_{B}\right)\right|$ and $l_{B} \cap F(i) \neq \emptyset$ for (at least) one agent $i \in l_{A}$, then $\min _{x \in l_{A}} r_{x}^{*}\left(l_{A}, l_{B}\right)=0$.

(4) For any $l_{B} \in L_{B}:$ (4.a) if $W_{A}\left(l_{B}\right) \neq \emptyset$ then $s_{A}^{*}\left(l_{B}\right) \in W_{A}\left(l_{B}\right)$; and (4.b) if $W_{A}\left(l_{B}\right)=\emptyset$ and $T\left(l_{B}\right) \neq \emptyset$ then $s_{A}^{*}\left(l_{B}\right) \in T\left(l_{B}\right)$.

(5) For any $l_{B} \in L_{B}:(5 . \mathrm{a})$ if $\left|J\left(s_{A}^{*}\left(l_{B}\right), l_{B}\right)\right| \geq\left|I\left(s_{A}^{*}\left(l_{B}\right), l_{B}\right)\right|$ then $r_{j}^{*}\left(l_{B}\right)=1$, for any $j \in l_{B}$; and (5.b) if $\left|J\left(s_{A}^{*}\left(l_{B}\right), l_{B}\right)\right|<\left|I\left(s_{A}^{*}\left(l_{B}\right), l_{B}\right)\right|$ and $s_{A}^{*}\left(l_{B}\right) \cap F(j) \neq \emptyset$ for (at least) one agent $j \in l_{B}$, then $\min _{y \in l_{B}} r_{y}^{*}\left(l_{B}\right)=0$.

(6) (6.a) If $\tilde{W}_{B} \neq \emptyset$ then $s_{B}^{*} \in \tilde{W}_{B}$; and (6.b) if $\tilde{W}_{B}=\emptyset$ and $\tilde{T} \neq \emptyset$ then $s_{B}^{*} \in \tilde{T}$.

The proof of Lemma 1 is shown in the Appendix. Conditions (1)-(2) determine the equilibrium behavior of citizens in the third stage (voting stage). Condition (1) says that, given two lists running for office, those agents that incur in the cross-cutting cost if chose to vote find it optimal to abstain. Condition (2) says that, given two lists running for office, the outcome of an election depends on the cardinalities of the sets $I\left(l_{A}, l_{B}\right)$ and $J\left(l_{A}, l_{B}\right)$, i.e., on the number of citizens for whom the voting decision does not imply a cost. Thus, in the case $\left|I\left(l_{A}, l_{B}\right)\right|>\left|J\left(l_{A}, l_{B}\right)\right|$, the agents in $I\left(l_{A}, l_{B}\right)$ can form a list $l_{A}$ that grants the office. For this equilibrium to hold, we need that more than $\left|J\left(l_{A}, l_{B}\right)\right|$ 
agents in the set $I\left(l_{A}, l_{B}\right)$ vote, so that no coalition of agents in $J\left(l_{A}, l_{B}\right)$ finds it profitable to deviate. Analogously, if $\left|I\left(l_{A}, l_{B}\right)\right|<\left|J\left(l_{A}, l_{B}\right)\right|$, the agents in $J\left(l_{A}, l_{B}\right)$ can form a list $l_{B}$ that grants the office. For this equilibrium to hold, we need that more than $\left|I\left(l_{A}, l_{B}\right)\right|$ agents in the set $J\left(l_{A}, l_{B}\right)$ vote. Finally, if $\left|I\left(l_{A}, l_{B}\right)\right|=\left|J\left(l_{A}, l_{B}\right)\right|$, the equilibrium requires that all the agents in $I\left(l_{A}, l_{B}\right)$ and $J\left(l_{A}, l_{B}\right)$ vote, which procures a tie. This is the only equilibrium, as in any other case, there will always be a coalition of agents, either $I\left(l_{A}, l_{B}\right)$ or $J\left(l_{A}, l_{B}\right)$, that will benefit from deviating and altering the outcome of the election.

Conditions (3) and (4) represent the equilibrium behavior in the second stage (response stage). Specifically, condition (3) determines the equilibrium behavior of agents in $N_{\mathcal{A}}$ regarding their acceptance decision. Thus, given a list $l_{B}$ (already formed) and a list $l_{A}$ (so far merely proposed), the equilibrium condition requires that, in view of (1)-(2), all the agents in $l_{A}$ accept to be in that list if they anticipate that their list will win or tie in an election. Differently, if they anticipate that their list will lose, the equilibrium condition requires that the list is not formed if there is at least one nominee susceptible to incur in cross-pressures. Condition (4) determines the equilibrium behavior of candidate $A$. This condition requires that candidate $A$, in view of (1)-(3), chooses $s_{A}^{*}\left(l_{B}\right) \in W_{A}\left(l_{B}\right)$ if the set of lists that allows her to get into office is not empty; and chooses $s_{A}^{*}\left(l_{B}\right) \in T\left(l_{B}\right)$ if such set is empty but the set of lists that allows her to tie is not.

Finally, conditions (5) and (6) represent the equilibrium behavior in the first stage (challenging stage). Specifically, condition (5) determines the equilibrium behavior of agents in $N_{\mathcal{B}}$ regarding their acceptance decision. Thus, given a list $l_{B}$, the equilibrium condition requires that, in view of (1)-(4), all the agents in $l_{B}$ accept to be in $l_{B}$ if they anticipate that either candidate $A$ will not be able to form a list, or she will but will not be able to win office with probability one. Differently, if they anticipate that their list will lose, the equilibrium condition requires that the list is not formed if there is at least one nominee susceptible to incur in cross-pressures. Finally, condition (6) determines the equilibrium behavior of candidate $B$. This condition requires that candidate $B$, in view of (1)-(5), chooses $s_{B}^{*} \in \tilde{W}_{B}$ if the set of lists that allows her to get into office is not empty; and chooses $s_{B}^{*} \in \tilde{T}$ if such set is empty but the set of lists that allows her to tie is not.

In the next proposition we show that the set of strong perfect equilibria is never empty (whatever $N_{A}, N_{B}, k$ and $g$ ) and that the outcome of any strong perfect equilibrium, in terms of which candidate, $A$ or $B$, gets into office, depends exclusively on the cardinalities of the sets $\tilde{W}_{B}$ and $\tilde{T}$.

Proposition $1 S^{*} \neq \emptyset$ and, for any $s^{*} \in S^{*}, P_{A}\left(s^{*}\right)= \begin{cases}0 & \text { if } \tilde{W}_{B} \neq \emptyset \\ \frac{1}{2} & \text { if } \tilde{W}_{B}=\emptyset \text { and } \tilde{T} \neq \emptyset \\ 1 & \text { otherwise. }\end{cases}$

The proof is shown in the appendix. From Proposition 1, we obtain the next corollary that states that all the strong perfect equilibria are outcome equivalent in terms of which alternative, $A$ or $B$, gets 
into office.

Corollary $1 P_{A}\left(s^{*}\right)=P_{A}\left(\tilde{s}^{*}\right)$ for any $\left(s^{*}, \tilde{s}^{*}\right) \in S^{*} \times S^{*}$.

The corollary follows directly from Proposition 1, since the sets $\tilde{W}_{B}$ and $\tilde{T}$ are defined independently of the strategy $s^{*} \in S^{*}$ under consideration.

In view of conditions (1)-(6) in Lemma 1, the set of strategy profiles that are strong perfect equilibria may be large. Note however that although this number may be large, all of the strong perfect equilibria are outcome equivalent in terms of which alternative gets into office (Corollary 1 ). This allows us to simplify the problem and restrict our attention to a particular subset of strong perfect equilibria that satisfies some reasonable conditions, ${ }^{11}$ it without restringing the set of the equilibrium outcomes.

Definition 5 Let $S^{* *} \subset S^{*}$ be the subset of strong perfect equilibria such that $s^{*} \in S^{* *}$ if and only if it satisfies the following conditions (7)-(11).

(7) For any $z \in N, v_{z}^{*}\left(l_{A}, l_{B}\right)= \begin{cases}1 & \text { if } z \in I\left(l_{A}, l_{B}\right) \cup J\left(l_{A}, l_{B}\right) \\ 0 & \text { otherwise. }\end{cases}$

(8) For any $i \in N_{\mathcal{A}}, r_{i}^{*}\left(l_{A}, l_{B}\right)= \begin{cases}1 & \text { if }\left|I\left(l_{A}, l_{B}\right)\right| \geq\left|J\left(l_{A}, l_{B}\right)\right| \\ 0 & \text { otherwise. }\end{cases}$

(9) $s_{A}^{*}\left(l_{B}\right) \in \arg \max _{l_{A} \in L_{A}}\left|I\left(l_{A}, l_{B}\right)\right|-\left|J\left(l_{A}, l_{B}\right)\right|$.

(10) For any $j \in N_{\mathcal{B}}, r_{j}^{*}\left(l_{B}\right)= \begin{cases}1 & \text { if }\left|J\left(s_{A}^{*}\left(l_{B}\right), l_{B}\right)\right| \geq\left|I\left(s_{A}^{*}\left(l_{B}\right), l_{B}\right)\right| \\ 0 & \text { otherwise. }\end{cases}$

(11) $s_{B}^{*} \in \arg \max _{l_{B} \in L_{B}}\left|J\left(s_{A}^{*}\left(l_{B}\right), l_{B}\right)\right|-\left|I\left(s_{A}^{*}\left(l_{B}\right), l_{B}\right)\right|$.

Note that we obtain conditions (7)-(11) by imposing some additional restrictions on conditions (1)-(6) in Lemma 1. In particular, we obtain (7) from conditions (1)-(2), once we require that all the agents in $I\left(l_{A}, l_{B}\right)$ and $J\left(l_{A}, l_{B}\right)$ vote. Note that for these citizens it is weakly dominant to vote. We obtain (8) from condition (3), if we consider that when a nominee in $l_{A}$ anticipates that his list will lose if formed, he refuses to be in the list (note that, in such a case, there may be agents in $l_{A}$ indifferent between accepting and not). ${ }^{12}$ We obtain (9) from condition (4) if we consider that, when candidate $A$ is able to win the election with two or more lists, she chooses one of the lists that grants her the maximum difference of votes (with respect to the number of votes of candidate $B$ ); and when candidate $A$ is neither

\footnotetext{
${ }^{11}$ As it is that citizens use weakly dominant strategies, and that candidates maximize (minimize) the difference of votes if this difference is favorable (unfavorable) to them.

${ }^{12}$ Note that the outcome induced by the behavior of citizens in this equilibrium is equivalent to a situation in which the candidates were allowed to decide whether to propose or not a list, and chose not to do it when anticipating no chances of winning.
} 
able to win nor to tie an election with any of her lists, she chooses one of the lists that grants her the minimum difference of votes (with respect to those of candidate $B$ ). We obtain (10) from condition (5) if, analogously to condition (8), we consider that when a citizen anticipates that his list $l_{B}$ will lose if formed, he refuses to be in that list (note that, in this case, there may also be agents in $l_{B}$ indifferent between accepting and not). Finally, we obtain (11) from condition (6) if, analogously to condition (9), we consider that, when candidate $B$ is able to win the election with two or more lists, she chooses one of the lists that grants her the maximum difference of votes; and when candidate $B$ is neither able to win nor to tie an election with any of her lists, she chooses one of the lists that grants her the minimum difference of votes.

The reader may note that for the set $S^{* *}$ of strong perfect equilibria that we consider, there are only three possible outcomes that we detail next: (i) The first possible outcome is that the incumbent, with the initial list $\widehat{l}_{A}$, keeps in office. This occurs when the challenger is not able to conform a list to threaten the incumbent, therefore no election takes place and the incumbent stays in power. (ii) The second possible outcome is that the challenger gets into office. This occurs when the incumbent is not able to conform a list to threaten the challenger. Here too, no election takes place and the challenger gets directly into office. (iii) Finally, the third possible outcome is a tie between the incumbent and the challenger. This occurs when the two candidates are able to conform and present a list, and then, an election takes place. Such election necessarily results in a tie, as otherwise the nominees in the loser list would have refused to be part of that list. Hence, in equilibrium, each alternative has necessarily a probability of one-half of getting into office.

\section{The candidates' game}

In the previous analysis we have fully characterized the behavior of citizens in equilibrium -conditions (7), (8) and (10). Regarding candidates, however, we just got the flavour of what they want to maximize -conditions (9) and (11). In this section we focus on the candidates' game, and study some features of the lists they choose in equilibrium. Then, we analyze the first mover advantage and study its implications for the equilibrium outcomes.

\subsection{Optimal lists}

Previous to this analysis, it is convenient to note that, in our model, all that matters is the structure of links among citizens in different groups, meaning that neither the voting decision nor the acceptance decision depends on the structure of links among citizens with the same preferred alternative. This implies that we can restrict our attention to the bipartite graph $\tilde{g}$ induced by the network $g$, which is represented by $\tilde{g}: N_{\mathcal{A}} \times N_{\mathcal{B}} \rightarrow\{0,1\}$, where $\tilde{g}(x, y)=g(x, y)$ for any $x \in N_{\mathcal{A}}$ and $y \in N_{\mathcal{B}}$. That is to 
say, we can focus on the structure of links (representing friendship) among citizens in different groups and abstract from the structure of these links among citizens within the same group.

Going into the analysis of the candidates' optimal behavior, we note that condition (7) determines that the only citizens voting in an election are those belonging to a list and those without cross-pressure. This implies that if the candidates want to win office, it is crucial for them to conform a list of citizens that generates as much pressure as possible on the opposite faction. As a result, it seems reasonable to think that a candidate would maximize her chances of winning by proposing a list whose nominees had as much friends in the other group as possible as, by so doing, she would generate cross pressure to the people in the other faction, who would optimally choose to abstain in the election in order to avoid the cross-cutting cost. This idea, however, does not always work, meaning that it is not always optimal for a candidate to choose the list that "covers" the maximum number of people in the other faction. More precisely, choosing this kind of list is optimal in the case the candidate has to nominate just one citizen $(k=1)$. Differently, if there are more than two seats in office for the winning alternative $(k \geq 2)$, this choice is not necessarily optimal. The reason is that, in such a case, it is important the number of people "covered" by a list, but it also matters the number of citizens "covered" by a list that runs for office, those we name running-citizens. The point is that, in equilibrium, the running-citizens will always vote for their most preferred ideology, regardless of whether they suffer from cross-pressures or not, and then, there is no point in inducing them such a cost.

Prior to the formal analysis of these ideas, we define three important concepts: the covering of a list, a list of maximal covering, and the set of covered nominees.

Definition 6 The covering of a list $l_{A} \in L_{A}$ of candidate $A$ which, with some abuse of notation, we denote by $F\left(l_{A}\right)$, consists on the subset of elements of $N_{\mathcal{B}}$ which are linked to at least one element of $l_{A}$, i.e., $F\left(l_{A}\right)=\bigcup_{i \in l_{A}} F(i)$. Analogously, the covering of a list $l_{B} \in L_{B}$ of candidate $B$ is $F\left(l_{B}\right)=\bigcup_{j \in l_{B}} F(j)$.

In other words, the covering of a list $l_{A}$ (resp. $l_{B}$ ) is the set of citizens supporting alternative $B$ (resp. $A$ ) who are friends of at least one citizen in the list $l_{A}$ (resp. $l_{B}$ ).

Definition 7 The set $l_{A}^{m c} \in L_{A}$ is a list of maximal covering for candidate $A$ if $F\left(l_{A}^{m c}\right)$ has maximal cardinality among the set of all possible lists $L_{A}$, i.e., $l_{A}^{m c} \in \arg \max _{l_{A} \in L_{A}}\left|F\left(l_{A}\right)\right|$. Analogously, the set $l_{B}^{m c} \in L_{B}$ is a list of maximal covering for candidate $B$ if $l_{B}^{m c} \in \arg \max _{l_{B} \in L_{B}}\left|F\left(l_{B}\right)\right|$. Let $L_{A}^{m c}$ and $L_{B}^{m c}$ be the sets of lists of maximal covering for candidates $A$ and $B$, respectively.

Definition 8 Given a pair of lists $\left(l_{A}, l_{B}\right) \in L_{A} \times L_{B}$, the set of covered nominees in the list $l_{A}$, which we denote by $C_{A}\left(l_{A}, l_{B}\right)$, is the set of citizens in $l_{A}$ who are linked to at least one citizen in $l_{B}$, i.e., $C_{A}\left(l_{A}, l_{B}\right)=l_{A} \cap F\left(l_{B}\right)$. Analogously, the set of covered nominees in the list $l_{B}$ is $C_{B}\left(l_{A}, l_{B}\right)=l_{B} \cap F\left(l_{A}\right)$.

That is to say, the set of covered nominees in a list is the set of citizens in that list that have at least one friend running for office for the cross-preferred alternative. 
We are now in position to analyze the equilibrium behavior of candidates, namely which is the candidates' optimal strategy. The next proposition characterizes this behavior.

Proposition 2 A strategy profile $s^{*} \in S^{* *}$ if and only if $s_{A}^{*}\left(l_{B}\right) \in \arg \max _{l_{A} \in L_{A}}\left|F\left(l_{A}\right)\right|+\left|C_{A}\left(l_{A}, l_{B}\right)\right|-$ $\left|C_{B}\left(l_{A}, l_{B}\right)\right|$ for any $l_{B} \in L_{B}, s_{B}^{*} \in \arg \max _{l_{B} \in L_{B}}\left|F\left(l_{B}\right)\right|-\left|F\left(s_{A}^{*}\left(l_{B}\right)\right)\right|+\left|C_{B}\left(s_{A}^{*}\left(l_{B}\right), l_{B}\right)\right|-\left|C_{A}\left(s_{A}^{*}\left(l_{B}\right), l_{B}\right)\right|$, and conditions (7), (8) and (10) hold. Additionally, if $s^{*} \in S^{* *}$, then $\left|F\left(l_{A}^{m c}\right)\right|-\left|F\left(s_{A}^{*}\left(l_{B}\right)\right)\right| \leq 2 k-2$ for any $l_{B} \in L_{B}$, and $\left|F\left(l_{B}^{m c}\right)\right|-\left|F\left(s_{B}^{*}\right)\right| \leq 2 k-2$.

The proof is shown in the appendix. This proposition contains two related ideas. The first one describes the equilibrium strategy for candidates, and shows that the optimal strategy takes into account not only the covering of a list, but also the difference between the nominees covered by the two lists. The second part of the proposition establishes an additional condition on the set of the equilibrium strategies. In particular, it sets up an upper limit on the difference in covering between the equilibrium strategy and the list of maximal covering, this upper limit being an increasing function in the number of citizens in a list $(k)$. Hence, the larger $k$, the greater the allowed difference in covering, and then, the larger the set of possible equilibrium strategies. As a result, the larger $k$, the more complex the problem become.

These two issues, however, turn out to be much more simple in the case $k=1$. In such a case, we obtain that any pair of lists of maximal covering constitutes a strong perfect equilibrium. This means that, in the case $k=1$, it is optimal for the candidates to nominate the citizen (or one of the citizens) with more friends in the other group. This further implies that the optimal strategy of a candidate is, in such a case, independent of the strategy of the opposing candidate. This idea is formalized in the next corollary.

Corollary 2 Consider $k=1$. A strategy profile $s^{*} \in S^{* *}$ if and only if $s_{A}^{*}\left(l_{B}\right) \in L_{A}^{m c}$ for any $l_{B} \in L_{B}$, $s_{B}^{*} \in L_{B}^{m c}$, and conditions (7), (8) and (10) hold.

The proof is shown in the appendix. The intuition for this result is that, in the case $k=1$, and for any pair of lists, the number of covered nominees in the two lists is equal, and then, all that matters is to propose the list with a maximal covering.

However, for the case of $k \geq 2$, this is not necessarily true. The reason is that, in such a case, the number of covered nominees in each of the two lists does not necessarily coincide. Hence, it may be the case that a list that is not a maximal covering procures a favorable difference of covered nominees that outweighs its smaller covering. As a result, it may be no equilibrium in maximal covering. To see this point in more detail, let us consider the network represented in Figure 1, where $N_{\mathcal{A}}=\left\{i_{1}, \ldots, i_{8}\right\}$, $N_{\mathcal{B}}=\left\{j_{1}, \ldots, j_{8}\right\}$, and the existence of a link between citizens $i$ and $j, \widetilde{g}(i, j)=1$, is represented by an arc between them. ${ }^{13}$

\footnotetext{
${ }^{13}$ Note that we restrict our attention to the bipartite graph $\widetilde{g}$ induced by $g$.
} 


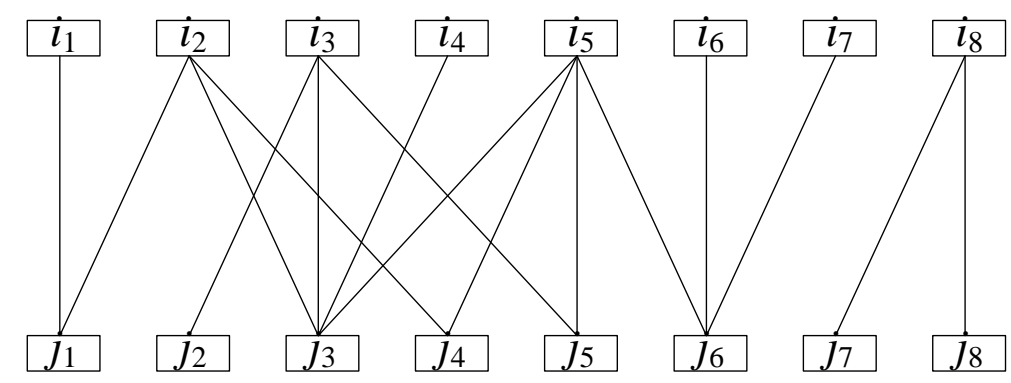

Figure 1.

Let us consider that there are three seats in office for the winning alternative, therefore $k=2$, and that candidate $A$, with a list $\widehat{l}_{A}$ of two citizens, is initially in power. In such a case, it is candidate $B$ that has to propose a list of two citizens to challenge the incumbent. We now obtain the covering of each citizen in $N_{\mathcal{A}}$ and the list of maximal covering for the incumbent.

$$
\begin{array}{llll}
F\left(i_{1}\right)=\left\{j_{1}\right\} & F\left(i_{3}\right)=\left\{j_{2}, j_{3}, j_{5}\right\} & F\left(i_{5}\right)=\left\{j_{3}, j_{4}, j_{5}, j_{6}\right\} & F\left(i_{7}\right)=\left\{j_{6}\right\} \\
F\left(i_{2}\right)=\left\{j_{1}, j_{3}, j_{4}\right\} & F\left(i_{4}\right)=\left\{j_{3}\right\} & F\left(i_{6}\right)=\left\{j_{6}\right\} & F\left(i_{8}\right)=\left\{j_{7}, j_{8}\right\}
\end{array}
$$

hence $l_{A}^{m c}=\left\{i_{5}, i_{8}\right\}$, with $F\left(l_{A}^{m c}\right)=F\left(i_{5}\right) \cup F\left(i_{8}\right)=\left\{j_{3}, j_{4}, j_{5}, j_{6}, j_{7}, j_{8}\right\}$, is the (unique) list of maximal covering of candidate $A$. We proceed analogously for candidate $B$ and obtain

$$
\begin{array}{llll}
F\left(j_{1}\right)=\left\{i_{1}, i_{2}\right\} & F\left(j_{3}\right)=\left\{i_{2}, i_{3}, i_{4}, i_{5}\right\} & F\left(j_{5}\right)=\left\{i_{3}, i_{5}\right\} & F\left(j_{7}\right)=\left\{i_{8}\right\} \\
F\left(j_{2}\right)=\left\{i_{3}\right\} & F\left(j_{4}\right)=\left\{i_{2}, i_{5}\right\} & F\left(j_{6}\right)=\left\{i_{5}, i_{6}, i_{7}\right\} & F\left(j_{8}\right)=\left\{i_{8}\right\}
\end{array}
$$

hence $l_{B}^{m c}=\left\{j_{3}, j_{6}\right\}$, with $F\left(l_{B}^{m c}\right)=F\left(j_{3}\right) \cup F\left(j_{6}\right)=\left\{i_{2}, i_{3}, i_{4}, i_{5}, i_{6}, i_{7}\right\}$, is the (unique) list of maximal covering of candidate $B$. As $k=2$ and $\left|F\left(l_{B}^{m c}\right)\right|=\left|F\left(l_{A}^{m c}\right)\right|=6$, by Proposition 2 we know that, for any $s^{*} \in S^{* *},\left|F\left(s_{B}^{*}\right)\right| \geq 4$ and $\left|F\left(s_{A}^{*}\left(s_{B}^{*}\right)\right)\right| \geq 4$.

We now show that there is no equilibrium in maximal covering, i.e., an equilibrium in which the two candidates propose their (unique) list of maximal covering. To see it, let us consider that the challenger proposes her list of maximal covering, $l_{B}^{m c}$, and that the incumbent does so, $l_{A}^{m c}$. In such a case, $\sum_{i \in N_{\mathcal{A}}} v_{i}^{*}\left(l_{A}^{m c}, l_{B}^{m c}\right)=3$ and $\sum_{j \in N_{\mathcal{B}}} v_{j}^{*}\left(l_{A}^{m c}, l_{B}^{m c}\right)=4$, i.e., the incumbent would not be able to maintain in office as the citizens proposed to be in her list would have anticipated the outcome of an election and would have optimally chosen not to run for office. If the incumbent, however, after observing $l_{B}^{m c}$, chooses to propose the list $l_{A}=\left\{i_{2}, i_{3}\right\}$, with $F\left(l_{A}\right)=F\left(i_{2}\right) \cup F\left(i_{3}\right)=\left\{j_{1}, j_{2}, j_{3}, j_{4}, j_{5}\right\}$, hence $\left|F\left(l_{A}\right)\right|<\left|F\left(l_{A}^{m c}\right)\right|$, she will guarantee a tie in the election as, in such a case, $\sum_{i \in N_{\mathcal{A}}} v_{i}^{*}\left(l_{A}, l_{B}^{m c}\right)=\sum_{j \in N_{\mathcal{B}}} v_{j}^{*}\left(l_{A}, l_{B}^{m c}\right)=4$. The reader may note, additionally, that neither the challenger nor the incumbent can do better than this, as for any of the lists of the challenger that provides a covering of five or six citizens, the incumbent can always respond with a list that guarantees a tie; and for any of the lists of the challenger that provides a covering of four, the incumbent can always beat her and win the election. Hence, the pair $l_{B}^{m c}=\left\{j_{3}, j_{6}\right\}$ and $l_{A}=\left\{i_{2}, i_{3}\right\}$ is an equilibrium of this game, but additionally, and more importantly, there is not an 
equilibrium in which the two candidates propose their list of maximal covering. We then conclude that, in the case $k=2$ (and, in general, for $k \geq 2$ ), there is not necessarily an equilibrium in maximal covering.

\subsection{The role of incumbency}

In this section we analyze if being the incumbent and, therefore, having the possibility to respond to the list proposed by the challenger, provides the former an advantage in order to get into office. As we shall see below, this is not the case when $k=1$ as, in such a case, the equilibrium outcome depends only on the network architecture, and not in the order of moves. Differently, we illustrate that when $k \geq 2$ there may exist a last mover advantage.

The next proposition shows that there is no advantage for the incumbent in the case $k=1$.

Proposition 3 Consider a population $N=N_{\mathcal{X}} \cup N_{\mathcal{Y}}$, where $N_{\mathcal{X}} \cap N_{\mathcal{Y}}=\emptyset$, and a bipartite graph defined by a function $\widetilde{g}: N_{\mathcal{X}} \times N_{\mathcal{Y}} \rightarrow\{0,1\}$. Let $S^{*}$ be the set of strong perfect equilibria of the game with $\mathcal{A}=\mathcal{X}$ and $\mathcal{B}=\mathcal{Y}$, and let $\hat{S}^{*}$ be the set of strong perfect equilibria of the game with $\mathcal{B}=\mathcal{X}$ and $\mathcal{A}=\mathcal{Y}$. If $k=1$, then $P_{A}\left(s^{*}\right)=P_{B}\left(\hat{s}^{*}\right)$ for any $s^{*} \in S^{*}$ and $\hat{s}^{*} \in \hat{S}^{*}$.

The proof is shown in the appendix. This result relies on Corollary 2 which implies that, in the case $k=1$, the outcome of an election is simply determined by the cardinalities of the sets $N_{\mathcal{A}}$ and $N_{\mathcal{B}}$, and by the covering of the lists proposed by the two candidates (specifically, the maximal covering lists). As a result, the probability of a candidate getting into office is, in equilibrium, independent of whether this candidate was initially in power or not. Or to say to it differently, there is no advantage of being the incumbent (last to move), but all that matters is the bipartite graph $\widetilde{g}$ induced by the network $g$.

This result, however, is no longer true when $k \geq 2$. In such a case, we know that the outcome of an election does not directly result from the covering of the two lists, but that the number of covered nominees is also an important variable. This implies that the incumbent, as the last player to move, has the possibility of placing her nominees within the group of covered citizens, and so recovering these voters (that otherwise would not have voted) and taking advantage of her position. To see this idea in more detail, assume $k \geq 2$ and consider a population $N=N_{\mathcal{X}} \cup N_{\mathcal{Y}}$, where $N_{\mathcal{X}} \cap N_{\mathcal{Y}}=\emptyset$, and a bipartite graph defined by a function $\widetilde{g}: N_{\mathcal{X}} \times N_{\mathcal{Y}} \rightarrow\{0,1\}$. There are three possibilities:

(i) Let us first consider the case $\mathcal{A}=\mathcal{X}$ and $\mathcal{B}=\mathcal{Y}$ with $\tilde{W}_{B} \neq \emptyset$. Then, in equilibrium, candidate $B$, by choosing $s_{B}^{*}=\bar{y} \in \tilde{W}_{B}$ gets into office, i.e., alternative $\mathcal{Y}$ is implemented in equilibrium. Then, in such a case, if we consider instead $\mathcal{B}=\mathcal{X}$ and $\mathcal{A}=\mathcal{Y}$, if candidate $A$ chooses $\hat{s}_{A}\left(l_{B}\right)=\bar{y}$ for any $l_{B} \in L_{B}$, then $I\left(\bar{y}, l_{B}\right)>J\left(\bar{y}, l_{B}\right)$ for any $l_{B} \in L_{B}$. Hence alternative $\mathcal{Y}$ is also implemented in equilibrium.

(ii) Let us now consider the case $\mathcal{A}=\mathcal{X}$ and $\mathcal{B}=\mathcal{Y}$ with $\tilde{W}_{B}=\emptyset$ and $\tilde{T} \neq \emptyset$. Then, in equilibrium, candidate $B$, by choosing $s_{B}^{*}=\bar{y} \in \tilde{T}$ gets a tie, i.e., alternative $\mathcal{Y}$ is implemented with probability 
of $1 / 2$. Then, in such a case, if we consider instead $\mathcal{B}=\mathcal{X}$ and $\mathcal{A}=\mathcal{Y}$, if candidate $A$ chooses $\hat{s}_{A}\left(l_{B}\right)=\bar{y}$ for any $l_{B} \in L_{B}$, then $I\left(\bar{y}, l_{B}\right) \geq J\left(\bar{y}, l_{B}\right)$ for any $l_{B} \in L_{B}$. Hence the (new) incumbent can guarantee a tie in the election, but it is still possible that she can win in equilibrium (alternative $\mathcal{Y}$ implemented with probability of 1$)$ if $W_{A}\left(l_{B}\right) \neq \emptyset$ for some $l_{B} \in L_{B}$.

(iii) Finally, consider the case $\mathcal{A}=\mathcal{X}$ and $\mathcal{B}=\mathcal{Y}$ with $\tilde{W}_{B}=\tilde{T}=\emptyset$. Then, in equilibrium, candidate $B$ does not get into office, i.e., alternative $\mathcal{Y}$ is not implemented in equilibrium. In such a case, if we consider instead $\mathcal{B}=\mathcal{X}$ and $\mathcal{A}=\mathcal{Y}$, it is possible that candidate $A$ gets a tie in the election (alternative $\mathcal{Y}$ implemented with probability of $1 / 2$ ) if $T\left(l_{B}\right) \neq \emptyset$ for some $l_{B} \in L_{B}$.

Hence, a candidate does never suffer from being the incumbent and, if $k \geq 2$, he may eventually benefit from it. To illustrate the incumbency advantage -cases (ii) and (iii) above-, let us consider the example represented in Figure 2, in which candidate $A$, with a list $\widehat{l}_{A}$ of two citizens (hence $k=2$ ), is initially in power. ${ }^{14}$

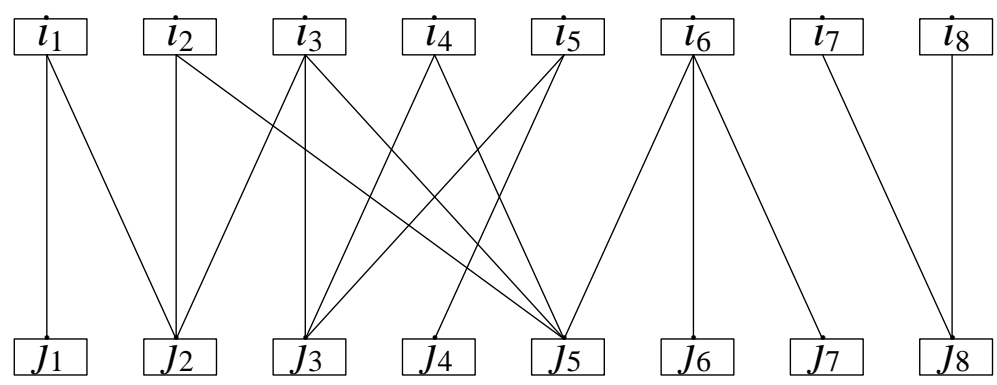

Figure 2.

Let us first suppose that $N_{\mathcal{A}}=\left\{i_{1}, \ldots, i_{8}\right\}$ and $N_{\mathcal{B}}=\left\{j_{1}, \ldots, j_{8}\right\}$. In such a case, it is optimal for candidate $B$ to propose her (unique) list of maximal covering, $s_{B}^{*}=l_{B}^{m c}=\left\{j_{5}, j_{8}\right\}$, with $F\left(l_{B}^{m c}\right)=$ $\left\{i_{2}, i_{3}, i_{4}, i_{6}, i_{7}, i_{8}\right\}$, and for candidate $A$, to respond to $l_{B}^{m c}$, with one of her lists of maximal covering. Specifically, $s_{A}^{*}\left(l_{B}^{m c}\right)=l_{A}^{m c}=\left\{i_{3}, i_{6}\right\}$, with $F\left(l_{A}^{m c}\right)=\left\{j_{2}, j_{3}, j_{5}, j_{6}, j_{7}\right\}$. If this is the case, then the election results in a tie, with $\sum_{i \in N_{\mathcal{A}}} v_{i}^{*}\left(s_{A}^{*}\left(l_{B}^{m c}\right), l_{B}^{m c}\right)=\sum_{j \in N_{\mathcal{B}}} v_{j}^{*}\left(s_{A}^{*}\left(l_{B}^{m c}\right), l_{B}^{m c}\right)=4$. Let us now consider the converse case, i.e., $N_{\mathcal{A}}=\left\{j_{1}, \ldots, j_{8}\right\}$ and $N_{\mathcal{B}}=\left\{i_{1}, \ldots, i_{8}\right\}$. In such a case, the new incumbent can always use her list of maximal covering, $l_{A}^{m c}=\left\{j_{5}, j_{8}\right\}$, to respond to the challenger's list, and by so doing, she can always guarantee a tie. However, in this new situation, the new incumbent has a strategy that allows her to win office. To see this, the reader may note that the new challenger, candidate $B$, is indifferent among proposing any of her lists, either of maximal covering or not, as in all the cases, the new incumbent, candidate $A$, wins the election by one vote. This means that, in the case the previous challenger were the incumbent, she could always do as well as when being the challenger. The reason for the incumbency advantage, as already pointed out, is that it is the second mover in this game that

\footnotetext{
${ }^{14}$ Note that the initial list $\hat{l}_{A}$ does not have any effect on the equilibrium strategies.
} 
has the advantage of placing her nominees within the group of covered citizens, and so gaining these voters that otherwise would not have voted. This possibility may be crucial, and in some cases, it may determine the outcome of an election.

This result, however, is not always true, namely it is not always the case that a candidate can always do strictly better by being the incumbent rather than the challenger. This ability, however, deeply depends on the network structure. For example, in the case of the network represented in Figure 1, the outcome of the election, i.e., the winner of the race, does not depend on who is the incumbent at that time. That is to say, an election, in such a case, always results in a tie independently on the identity of the candidate initially in power.

Finally, working a bit more on these examples, we observe that, for a given network $g$, if we were to repeat the game for a number of times, we would obtain three well distinguished outcomes: (i) The first one is that the incumbent initially in power perpetuates in office. This will occur if the network $g$ is such that the challenger does not have any chances of getting into office. (ii) The second one is the example presented in Figure 1 above, in which an election always results in a tie independently on who is the incumbent at that time. In such a case, we will have a random rotation in power between the two candidates. (iii) The third one is the example presented in Figure 2 above, in which the network is such that the incumbent at the beginning of the game is weaker than the challenger but she has the incumbency advantage. In such a case, the first election will result in a tie that could yield any of the two candidates into office. Now, if the challenger were to get into office at the first election, she would maintain in power for the rest of the game (unless the network changes). Differently, if the incumbent were to keep in office after the first election, the next election would also result in a tie, and we would be in the previous case. Summing up, any election in this case will result in a tie until the challenger gets into office; and from then on, the new incumbent, i.e., the former challenger, stays in power.

\section{Analysis for extreme values of the cross-cutting cost}

This section deals with the more extreme cases of a low $(c<d<h)$ and a high $(c>h>d)$ cross-cutting cost. Here we show that for a low cross-cutting cost, only under very limited conditions a strong perfect equilibrium exists. Regarding the case of high cross-pressures, we observe that the existence of such high costs favors the challenger as compared to the case of an intermediate cost $(h>c>d)$ analyzed throughout the paper.

\subsection{Low cross-cutting cost}

We first consider the case of a low cross-cutting cost, $c<d<h$. Here, we differentiate two cases: $c>\frac{d}{2}$ and $c<\frac{d}{2}$, that we study separately. 
We first analyze the case $c>\frac{d}{2}$. The next proposition characterizes the conditions for equilibrium existence.

Proposition 4 Assume $h>d>c>\frac{d}{2}$. Then $S^{*} \neq \emptyset$ if and only if, for any $\left(l_{A}, l_{B}\right) \in L_{A} \times L_{B}$, one of the following three conditions (I)-(III) hold. (I) $\left|I\left(l_{A}, l_{B}\right)\right|=\left|J\left(l_{A}, l_{B}\right)\right| ;(I I)\left|I\left(l_{A}, l_{B}\right)\right| \geq\left|N_{\mathcal{B}}\right| ;$ (III) $\left|J\left(l_{A}, l_{B}\right)\right| \geq\left|N_{\mathcal{A}}\right|$.

The proof is shown in the appendix. This result relies on the fact that, given $\frac{d}{2}<c<d$ : (i) A citizen who is abstaining and whose preferred alternative looses the election, finds it profitable to deviate to vote, and so, to incur in the cross-cutting cost, if and only if, by participating in a deviating coalition, his preferred alternative turns into the winner; (ii) A citizen who is voting and incurring in cross-pressure, and whose preferred alternative wins the election, finds it profitable to deviate to abstain, and so, to save the cross-cutting cost, if and only if after his deviation his preferred alternative either still wins or ties in the election; (iii) A citizen who is voting and incurring in cross-pressures, and whose preferred alternative ties in the election, finds it always profitable to deviate to abstain, and so, to save the cross-cutting cost.

$>$ From Proposition 4 we learn that only under very limited conditions a strong perfect equilibrium (in pure strategies) can be found. In particular, for an equilibrium to exist we need that either the number of citizens whose voting decision does not imply a cost is the same in the two groups, in which case the equilibrium will result in a tie; or that the number of this people in one of these groups is higher or equal than the total number of citizens in the other faction, in which case the larger faction wins office. Clearly, to require that one of these three conditions hold for each pair of list is quite strong, and so, an equilibrium fails to exist in most of the cases.

We now analyze the case $c<\frac{d}{2}$. The next proposition characterizes the conditions for equilibrium existence.

Proposition 5 Assume $h>\frac{d}{2}>c$. Then $S^{*} \neq \emptyset$ if and only if one of the following three conditions (I')(III') hold. (I') $\left|N_{\mathcal{A}}\right|=\left|N_{\mathcal{B}}\right|$; (II') $\left|I\left(l_{A}, l_{B}\right)\right|>\left|N_{\mathcal{B}}\right|$ for any $\left(l_{A}, l_{B}\right) \in L_{A} \times L_{B} ;\left(I I I^{\prime}\right)\left|J\left(l_{A}, l_{B}\right)\right|>\left|N_{\mathcal{A}}\right|$ for any $\left(l_{A}, l_{B}\right) \in L_{A} \times L_{B}$.

The proof is omitted, since it is very similar to the proof of Proposition 4. The new condition $c<\frac{d}{2}$ implies that a citizen who is not voting and whose preferred alternative is either losing or tying, is always willing to deviate to vote, even in the case it implies to incur in the cross-cutting cost, if by so doing, his preferred alternative turns either from losing to tying, or from tying to winning. Additionally, the cross-cutting cost is in this case so small that no citizen finds it profitable to deviate from voting to abstaining if, with his deviation, the probability that his preferred alternative wins office is reduced (even if by abstaining this citizen saves the cross-cutting cost).

The reader may note, that although restrictive, these conditions are no such strong as the ones in Proposition 4. Specifically, condition (I') says that for networks with factions of equal size, there is always 
a strong perfect equilibrium in which candidates propose any pair of lists, all the nominees accept to be in the lists, and all the citizens vote. This equilibria always results in a tie. Differently, for the cases in which $\left|N_{\mathcal{A}}\right| \neq\left|N_{\mathcal{B}}\right|$, the equilibrium requirements are strong, as in the previous case and so, an equilibrium fails to exist in most of the cases. Note that, if the requirements are satisfied, the equilibrium always results in the larger faction winning office.

\subsection{High cross-cutting cost}

We now consider the case of a high cross-cutting cost, $c>h>d$. This parameter configuration says that cross-pressures are so important that even the benefit of holding office (itself) does not pay the cross-cutting cost. Here, we differentiate two cases: $c>h+d$ and $h+d>c$, that we study separately.

Let us first consider the case $c>h+d$. In such a case, the main point is that in any strong perfect equilibrium, none of the citizens in the incumbent's faction will find it profitable to run for office if they have at least one friend in the challenger's list. This is so even if, by accepting, they anticipate that they will win or tie the election. Formally, $r_{i}^{*}\left(l_{B}\right)=0$ for any $i \in N_{A}$ such that $l_{B} \cap F(i) \neq \emptyset$. This implies that, in any equilibrium, and for a given list $l_{B}$, the incumbent is "restricted" to choose the members of her list $s_{A}^{*}\left(l_{B}\right)$ from the set $N_{A} \backslash F\left(l_{B}\right)$. This further implies that, in equilibrium, the set of covered nominees for any of the two sides is empty. This fact rules out the incumbency advantage that we observed in Section 4.2 for $k \geq 2$, when $h>2 c-d$ and $c>d$. Moreover, in the current case, there would be a challenger's advantage, since the set of "available" lists for the incumbent is restricted by the challenger's list.

We finally consider the case $c<h+d$. The main difference with respect to the previous case is that, when $c<h+d$, a citizen in the incumbent's faction finds it profitable to run for office if he anticipates that his list will win the election, regardless of whether he incurs in cross-pressures or not. Note that he will not accept in the case that he anticipates that his list will tie or loose in the election. As a result, the challenger can still impose a restriction (although weaker than in the previous case) on the incumbent's lists, as none of the covered citizens will find it profitable to run for office unless they anticipate that they will win for sure. Or, to say it differently, the incumbent partially conserves her last mover advantage observed in Section 4.2 (recall she can strategically place her nominees within the set of covered citizens, and so, recover these votes), but only in the case this advantage allows her to win (in such a case, a covered citizen in $N_{A}$ still finds it profitable to run for the incumbent). Differently, she can no longer strategically place her nominees within the set of covered citizens when, by so doing, she is, at most, able to tie (in such a case, no covered citizen will be willing to run for office in her list). Hence, it is not clear which position, i.e., being the incumbent or the challenger, is more favorable in order to get into office, which depends on the network architecture. 


\section{Conclusion}

From the forties to the sixties, a number of studies by social psychologists and political sociologists (Lazarsfeld et al., (1944), Simmel (1955) or Campbell et al. (1960) among others) suggested that people experiencing some degree of conflict among the factors swaying their voting decision tend to participate less in politics. More recently, Mutz $(2002,2006)$, in an empirical study for the US presidential and congressional elections of the 1992 and 1996, finds new and recent evidence showing that people whose networks involve greater political disagreement are less likely to participate in politics.

Our paper is an attempt to incorporate this well documented fact into a model of voter turnout, to the aim of studying how the existence of cross-pressures may shape voting decisions, and so, political outcomes; and how political parties may exploit this effect to their interest. To this aim we propose a model of elections in small networks where the structure of friendships is common knowledge, and so the existence of cross-pressures attaches a special relevance.

From a technical point of view, we characterize the set of strong perfect equilibria of the game and to show that they all are outcome equivalent in terms of which alternative gets into office. This means that, given a network and a particular configuration of the parameters, in all of the strong perfect equilibria, the candidate getting into office is always the same. Regarding the candidates' optimal strategy, we establish the conditions that an optimal strategy must satisfy, and determine a lower bound for the covering of an optimal list, which is linear in the number of seats in office. The optimal strategy is, in general, difficult to identify as it deeply depends on the network architecture. Nevertheless, we show that for the case in which each candidate has to propose just one citizen, a candidate's optimal list belongs to the set of lists of maximal covering. This result, however, does not necessarily hold when candidates have to propose lists of two or more citizens. In such a case, an equilibrium in maximal covering does not necessarily exist. We also derive some implications on the role of incumbency. In particular, we observe that the incumbent, as the last player to move, may benefit from this position and so enjoys an incumbency advantage (which does not exist in the case of candidates making lists of just one citizen). Finally, we explore the equilibria of the game for extreme values of the cross-cutting cost. We observe that, for low values of the cross-cutting cost, an equilibrium may generally fail to exist. On the other hand, for high values of cross-pressures we observe that the existence of such high costs favors the challenger as compared to the case of an intermediate cost.

From a more meaningful point of view, we bring into an strategic analysis of agents' behavior the idea that cross-pressures may be relevant for voting games. We propose a stylized model of elections in small networks, which serves to derive some initial insights into the issue, as well as to point out some of the problems that an analysis of cross-pressures in small networks may face. Nevertheless, this is a first attempt to model a game with cross-pressure considerations, and as such, there is much to do on this respect. For example, it would be interesting to take a more realistic approach for the cross-cutting 
cost and to allow for different levels of cross-pressures; or to make the cost dependent on the relative number of non-affine friends over the total number of social contacts. For such set-ups, the analysis of the strategic considerations that the existence of cross-pressures implies for voting games is something still unexplored, that we plan to address in future work.

\section{Appendix}

\section{Proof of Lemma 1}

The proof is divided in two parts. In the first part, we show that conditions (1)-(6) are necessary for a strategy profile $s^{*}$ to be a strong perfect equilibrium and in the second part we show that they are also sufficient.

\section{Part I: necessary conditions.}

We first prove that conditions (1)-(6) are necessary for a strategy profile $s^{*}$ to be a strong perfect equilibrium. To this aim, we follow a backwards induction argument (allowing for the deviations of all possible coalitions at every subgame). We start considering the third stage. Given a pair of lists $l_{B}$ and $l_{A}$, and given the assumption $c>d>0$, it is strictly dominated for any agent $x \in N \backslash\left(I\left(l_{A}, l_{B}\right) \cup J\left(l_{A}, l_{B}\right)\right)$ to choose $v_{x}^{*}\left(l_{A}, l_{B}\right)=1$. Hence, condition (1) is necessary for $s^{*}$ to be a strong perfect equilibrium.

Given a pair of lists $l_{B}$ and $l_{A}$, by condition (1) we know that the outcome of the election will be determined by the votes of the citizens in the sets $I\left(l_{A}, l_{B}\right)$ and $J\left(l_{A}, l_{B}\right)$. For each of the citizen in these sets it is weakly dominant to vote, since they either incur or do not incur in the cross-cutting cost, regardless of their decision in the voting stage.

We first claim that if $\left|I\left(l_{A}, l_{B}\right)\right|=\left|J\left(l_{A}, l_{B}\right)\right|$, then $v_{x}^{*}\left(l_{A}, l_{B}\right)=1$ for any $x \in I\left(l_{A}, l_{B}\right) \cup J\left(l_{A}, l_{B}\right)$ is a necessary condition for $s^{*}$ to be a strong perfect equilibrium. For the sake of contradiction, assume it is not the case. Then, if $\sum_{i \in N_{\mathcal{A}}} v_{i}^{*}\left(l_{A}, l_{B}\right)<\sum_{j \in N_{\mathcal{B}}} v_{j}^{*}\left(l_{A}, l_{B}\right)$, a coalition formed by those individuals in $I\left(l_{A}, l_{B}\right)$ who do not vote would find it profitable to deviate to vote and make their preferred alternative either to tie or to win the election, a contradiction. Analogously, if $\sum_{i \in N_{\mathcal{A}}} v_{i}^{*}\left(l_{A}, l_{B}\right)>\sum_{j \in N_{\mathcal{B}}} v_{j}^{*}\left(l_{A}, l_{B}\right)$, then a coalition formed by those individuals in $J\left(l_{A}, l_{B}\right)$ who do not vote would find it profitable to deviate to vote and make their preferred alternative either to tie or to win the election, a contradiction. Finally, if $\sum_{i \in N_{\mathcal{A}}} v_{i}^{*}\left(l_{A}, l_{B}\right)=\sum_{j \in N_{\mathcal{B}}} v_{j}^{*}\left(l_{A}, l_{B}\right)<\left|I\left(l_{A}, l_{B}\right)\right|$, then any individual in $I\left(l_{A}, l_{B}\right) \cup J\left(l_{A}, l_{B}\right)$ who does not vote would find it profitable to deviate to vote and make his preferred alternative to win the election, a contradiction. This proves the claim.

We now claim that if $\left|I\left(l_{A}, l_{B}\right)\right|>\left|J\left(l_{A}, l_{B}\right)\right|$ then $\sum_{i \in N_{\mathcal{A}}} v_{i}^{*}\left(l_{A}, l_{B}\right)>\left|J\left(l_{A}, l_{B}\right)\right|$ is a necessary condition for $s^{*}$ to be a strong perfect equilibrium. For the sake of contradiction, assume it is not the case. Then, if $\sum_{i \in N_{\mathcal{A}}} v_{i}^{*}\left(l_{A}, l_{B}\right) \leq \sum_{j \in N_{\mathcal{B}}} v_{j}^{*}\left(l_{A}, l_{B}\right)$, a coalition formed by those individuals in $I\left(l_{A}, l_{B}\right)$ who do not vote would find it profitable to deviate to vote and make their preferred alternative to win 
the election, a contradiction. Finally, if $\sum_{i \in N_{\mathcal{A}}} v_{i}^{*}\left(l_{A}, l_{B}\right)>\sum_{j \in N_{\mathcal{B}}} v_{j}^{*}\left(l_{A}, l_{B}\right)$, then a coalition formed by those individuals in $J\left(l_{A}, l_{B}\right)$ who do not vote would find it profitable to deviate to vote and make their preferred alternative either to tie or to win the election, a contradiction. This proves the claim. Analogously, it can be shown that, if $\left|J\left(l_{A}, l_{B}\right)\right|>\left|I\left(l_{A}, l_{B}\right)\right|$, then $\sum_{j \in N_{\mathcal{B}}} v_{j}^{*}\left(l_{A}, l_{B}\right)>\left|I\left(l_{A}, l_{B}\right)\right|$ is a necessary condition for $s^{*}$ to be a strong perfect equilibrium. Hence, condition (2) is necessary for $s^{*}$ to be a strong perfect equilibrium.

Now, given that the equilibrium behavior in the third stage is determined by conditions (1) and (2), we analyze the equilibrium behavior in the second stage. Given a list $l_{B} \in L_{B}$ (already formed) and a list $l_{A} \in L_{A}$ (so far merely proposed), we first claim that if $\left|I\left(l_{A}, l_{B}\right)\right| \geq\left|J\left(l_{A}, l_{B}\right)\right|$ then $r_{i}^{*}\left(l_{A}, l_{B}\right)=1$, for any $i \in l_{A}$, is a necessary condition for $s^{*}$ to be a strong perfect equilibrium. For the sake of contradiction, assume that $r_{i}^{*}\left(l_{A}, l_{B}\right)=0$ for at least one agent $i \in l_{A}$. Then the list $l_{A}$ does not form and the list $l_{B}$ gets into office. If the coalition formed by all the agents in $l_{A}$ who do not accept deviated to accept, the list would form and, given (1), (2) and $\left|I\left(l_{A}, l_{B}\right)\right| \geq\left|J\left(l_{A}, l_{B}\right)\right|$, it would either tie or win the election. Hence, given the assumption $h>2 c-d$, the coalition does have incentives to deviate, a contradiction. We now claim that if $\left|I\left(l_{A}, l_{B}\right)\right|<\left|J\left(l_{A}, l_{B}\right)\right|$ and $l_{B} \cap F(i) \neq \emptyset$ for (at least) one agent $i \in l_{A}$, then it is necessary for $s^{*}$ to be a strong perfect equilibrium that $r_{x}^{*}\left(l_{A}, l_{B}\right)=0$ for (at least) one agent $x \in l_{A}$. For the sake of contradiction, assume $\left|I\left(l_{A}, l_{B}\right)\right|<\left|J\left(l_{A}, l_{B}\right)\right|$ and $l_{B} \cap F(i) \neq \emptyset$ for (at least) one agent $i \in l_{A}$ and $r_{x}^{*}\left(l_{A}, l_{B}\right)=1$ for all $x \in l_{A}$. Then, the list $l_{A}$ forms and, given (1) and (2), list $l_{B}$ gets into office and agent $i$ 's payoff is $-c$, since $i \in l_{A}$ and $l_{B} \cap F(i) \neq \emptyset$, whereas if he deviated to choose $r_{i}^{*}\left(l_{A}, l_{B}\right)=0$, list $l_{B}$ would also get into office but agent $i$ would avoid the cross-cutting cost and get a payoff $0>-c$, a contradiction with $s^{*}$ being a strong perfect equilibrium. This proves the claim. Hence, condition (3) is necessary for $s^{*}$ to be a strong perfect equilibrium.

Now, given (1), (2) and (3), we analyze the equilibrium behavior of candidate $A$. Given a list $l_{B} \in L_{B}$ (already formed), by (1), (2), and (3), if candidate $A$ chooses a list $s_{A}^{*}\left(l_{B}\right) \in W_{A}\left(l_{B}\right)$ she gets into office and achieves a payoff $h+d$; whereas if $s_{A}^{*}\left(l_{B}\right) \notin W_{A}\left(l_{B}\right)$ she, at most, gets a tie (being her payoff at most $\left.\frac{h+d}{2}\right)$. Hence, if $W_{A}\left(l_{B}\right) \neq \emptyset$ then $s_{A}^{*}\left(l_{B}\right) \in W_{A}\left(l_{B}\right)$ is a necessary condition for $s^{*}$ to be a strong perfect equilibrium. In the case $W_{A}\left(l_{B}\right)=\emptyset$, by choosing a list $s_{A}^{*}\left(l_{B}\right) \in T\left(l_{B}\right)$ there is a tie in the election (being candidate $A$ 's payoff $\frac{h+d}{2}$ ) whereas if $s_{A}^{*}\left(l_{B}\right) \notin T\left(l_{B}\right)$ list $l_{B}$ gets into office (being candidate $A$ 's payoff 0$)$. Hence, if $W_{A}\left(l_{B}\right)=\emptyset$ and $T\left(l_{B}\right) \neq \emptyset$ then $s_{A}^{*}\left(l_{B}\right) \in T\left(l_{B}\right)$ is a necessary condition for $s^{*}$ to be a strong perfect equilibrium. Hence, condition (4) is necessary for $s^{*}$ to be a strong perfect equilibrium.

Now, given that the equilibrium behavior in the second and third stage is determined by conditions (1)-(4), we analyze the equilibrium behavior in the first stage. Given a list $l_{B}$ (so far merely proposed), we first claim that if $\left|J\left(s_{A}^{*}\left(l_{B}\right), l_{B}\right)\right| \geq\left|I\left(s_{A}^{*}\left(l_{B}\right), l_{B}\right)\right|$ then $r_{j}^{*}\left(l_{B}\right)=1$, for any $j \in l_{B}$, is a necessary condition for $s^{*}$ to be a strong perfect equilibrium. For the sake of contradiction, assume that $r_{j}^{*}\left(l_{B}\right)=0$ for at least one agent $j \in l_{B}$. Then the list $l_{B}$ does not form and the list $\hat{l}_{A}$ remains in office. However, 
if the coalition formed by all the agents in $l_{B}$ who do not accept deviated to accept, the list would form and, given (1)-(4) and $\left|J\left(s_{A}^{*}\left(l_{B}\right), l_{B}\right)\right| \geq\left|I\left(s_{A}^{*}\left(l_{B}\right), l_{B}\right)\right|$, it would either tie or win the election. Given the assumption $h>2 c-d$, the coalition has incentives to deviate, a contradiction. We now claim that if $\left|J\left(s_{A}^{*}\left(l_{B}\right), l_{B}\right)\right|<\left|I\left(s_{A}^{*}\left(l_{B}\right), l_{B}\right)\right|$ and $s_{A}^{*}\left(l_{B}\right) \cap F(j) \neq \emptyset$ for (at least) one agent $j \in l_{B}$, then it is necessary for $s^{*}$ to be a strong perfect equilibrium that $r_{y}^{*}\left(l_{B}\right)=0$ for (at least) one agent $y \in l_{B}$. For the sake of contradiction, assume $\left|J\left(s_{A}^{*}\left(l_{B}\right), l_{B}\right)\right|<\left|I\left(s_{A}^{*}\left(l_{B}\right), l_{B}\right)\right|$ and $s_{A}^{*}\left(l_{B}\right) \cap F(j) \neq \emptyset$ for (at least) one agent $j \in l_{B}$ and $r_{y}^{*}\left(l_{B}\right)=1$ for all $y \in l_{B}$. Then, the list $l_{B}$ forms and, given (1)-(4), the list $s_{A}^{*}\left(l_{B}\right)$ forms and gets into office, and agent $j$ 's payoff is $-c$, since $j \in l_{B}$ and $s_{A}^{*}\left(l_{B}\right) \cap F(j) \neq \emptyset$, whereas if he deviated to choose $r_{j}^{*}\left(l_{B}\right)=0$, list $\hat{l}_{A}$ would remain in office and agent $j$ would avoid the cross-cutting cost and get a payoff $0>-c$, a contradiction with $s^{*}$ being a strong perfect equilibrium. This proves the claim. Hence, condition (5) is necessary for $s^{*}$ to be a strong perfect equilibrium.

Now, given (1)-(5) we analyze the equilibrium behavior of candidate $B$. By (1)-(5), if candidate $B$ chooses a list $s_{B}^{*} \in \tilde{W}_{B}$ she gets into office and achieves a payoff $h+d$; whereas if $s_{B}^{*} \notin \tilde{W}_{B}$ she, at most, gets a tie (being her payoff at most $\frac{h+d}{2}$ ). Hence, if $\tilde{W}_{B} \neq \emptyset$ then $s_{B}^{*} \in \tilde{W}_{B}$ is a necessary condition for $s^{*}$ to be a strong perfect equilibrium. In the case $\tilde{W}_{B}=\emptyset$, by choosing a list $s_{B}^{*} \in \tilde{T}$ there is a tie in the election (being candidate $B$ 's payoff $\frac{h+d}{2}$ ) whereas if $s_{B}^{*} \notin \tilde{T}$ alternative $\mathcal{A}$ gets into office (being candidate $B$ 's payoff 0$)$. Hence, if $\tilde{W}_{B}=\emptyset$ and $\tilde{T} \neq \emptyset$ then $s_{B}^{*} \in \tilde{T}$ is a necessary condition for $s^{*}$ to be a strong perfect equilibrium. Hence, condition (6) is necessary for $s^{*}$ to be a strong perfect equilibrium.

\section{Part II: sufficient conditions.}

Once we have proven that (1)-(6) are necessary conditions for a strategy profile $s^{*}$ to be a strong perfect equilibrium, we prove that they are also sufficient, i.e., we claim that, if a strategy profile $s^{*} \in S$ satisfies (1)-(6), then $s^{*}$ is a strong perfect equilibrium. We have to prove that, if $s^{*}$ satisfies (1)-(6), no coalition of players has incentives to deviate at any proper subgame. We also use a backwards induction argument. In the third stage of the game, for any possible pair of proposed lists $\left(l_{A}, l_{B}\right) \in L_{A} \times L_{B}$, given (1) and (2), the outcome of the election is determined by the cardinalities of the sets $I\left(l_{A}, l_{B}\right)$ and $J\left(l_{A}, l_{B}\right)$. If $\left|I\left(l_{A}, l_{B}\right)\right|>\left|J\left(l_{A}, l_{B}\right)\right|$, list $l_{A}$ gets into office and no individual in $N_{\mathcal{A}}$ has incentives to be part of a deviating coalition. The outcome of the election can only be altered if individuals in the set $N_{\mathcal{B}} \backslash J\left(l_{A}, l_{B}\right)$ deviate to vote but, as we already noted, it is strictly dominated to vote for such individuals. Hence, no coalition has incentives to deviate in the voting stage when $\left|I\left(l_{A}, l_{B}\right)\right|>\left|J\left(l_{A}, l_{B}\right)\right|$. By an analogous reasoning we can also conclude that no coalition of citizens has incentives to deviate in the third stage when $\left|J\left(l_{A}, l_{B}\right)\right|>\left|I\left(l_{A}, l_{B}\right)\right|$. Finally if $\left|I\left(l_{A}, l_{B}\right)\right|=\left|J\left(l_{A}, l_{B}\right)\right|$ the outcome of the election is a tie. Clearly, no individual in the sets $I\left(l_{A}, l_{B}\right)$ and $J\left(l_{A}, l_{B}\right)$ has incentives to be part of a deviating coalition, since by deviating to not voting such individual can only reduce the probability that his preferred alternative gets into office. On the other hand, since for the remaining citizens it is strictly dominated to vote, we conclude that no coalition has incentives to deviate in the voting stage 
when $\left|I\left(l_{A}, l_{B}\right)\right|=\left|J\left(l_{A}, l_{B}\right)\right|$.

We now show that, given a list $l_{B}$ (already formed) and a list $l_{A}$ (so far merely proposed), if $s^{*}$ satisfies (1)-(6) then no coalition of players in $l_{A}$ has incentives to deviate (in the acceptance decisions). If $\left|I\left(l_{A}, l_{B}\right)\right| \geq\left|J\left(l_{A}, l_{B}\right)\right|$, given (1)-(3), $l_{A}$ gets into power and, if any coalition of players in $l_{A}$ deviates, then alternative $l_{B}$ gets into power instead. Given the assumption $h>2 c-d$, no coalition of players deviates at this stage when $\left|I\left(l_{A}, l_{B}\right)\right| \geq\left|J\left(l_{A}, l_{B}\right)\right|$. On the other hand, if $\left|I\left(l_{A}, l_{B}\right)\right|<\left|J\left(l_{A}, l_{B}\right)\right|$, given (1)-(3), $l_{B}$ gets into office and it cannot be prevented by the deviation of any coalition of players in $l_{A}$ in their acceptance decisions. Note that condition (3.b) requires that in such a case, and given $l_{B}$, the list $l_{A}$ may form only if no player in the list is susceptible to incur in the cross-cutting cost. Hence, a deviation at this stage cannot improve the payoff of any agent in $l_{A}$ but can reduce the payoff of some of them (those who could incur in the cross-cutting cost $c$ if the list were formed). Hence no coalition of players in $l_{A}$ has incentives to deviate at this stage. Note also that, given (1)-(3), candidate $A$ does not have incentives to deviate from the behavior prescribed by (4), since it prescribes to choose a list that allows her to get into office, when such a list exists; and to choose a list that allows her to tie in the case there is no list susceptible to win but there exists at least one list that can tie.

We finally show that, if $s^{*}$ satisfies (1)-(6), then there is no profitable deviation in the first stage. We first show that given a list $l_{B}$ so far merely proposed, if $s^{*}$ satisfies (1)-(6), then no coalition of players in $l_{B}$ has incentives to deviate (in the acceptance decisions). If $\left|J\left(s_{A}^{*}\left(l_{B}\right), l_{B}\right)\right| \geq\left|I\left(s_{A}^{*}\left(l_{B}\right), l_{B}\right)\right|$, given (1)-(5), $l_{B}$ gets into power and, if any coalition of players in $l_{B}$ deviates, then $s_{A}^{*}\left(l_{B}\right)$ wins office instead. Given the assumption $h>2 c-d$, no coalition of players deviates at this stage when $\left|J\left(s_{A}^{*}\left(l_{B}\right), l_{B}\right)\right| \geq\left|I\left(s_{A}^{*}\left(l_{B}\right), l_{B}\right)\right|$. On the other hand, if $\left|J\left(s_{A}^{*}\left(l_{B}\right), l_{B}\right)\right|<\left|I\left(s_{A}^{*}\left(l_{B}\right), l_{B}\right)\right|$, given (1)-(5), either $\hat{l}_{A}$ or $s_{A}^{*}\left(l_{B}\right)$ gets into office and it cannot be prevented by the deviation of any coalition of players in $l_{B}$ in their acceptance decisions. Note that condition (5.b) requires that, in such a case, the list $l_{B}$ may form only if no player in the list is susceptible to incur in the cross-cutting cost. Hence, a deviation at this stage cannot improve the payoff of any agent in $l_{B}$ but can reduce the payoff of some of them (those who could incur in the cross-cutting cost $c$ if the list were formed). Hence no coalition of players in $l_{B}$ has incentives to deviate at this stage. Note also that, given (1)-(5), candidate $B$ does not have incentives to deviate from the behavior prescribed by (6), since it prescribes to choose a list that allows her to get into office, when such a list exists; and to choose a list that allows her to tie in the case there is no list susceptible to win but there exists at least one list that can tie. This completes the proof.

\section{Proof of Proposition 1}

The fact that $S^{*} \neq \emptyset$ follows directly from Lemma 1 since, by construction, the set of strategies satisfying conditions (1)-(6) is not empty. Additionally, for any strategy profile $s^{*} \in S^{*}$ satisfying conditions (1)-(6), we have three possibilities:

(i) $\tilde{W}_{B} \neq \emptyset$. In such a case we know, by $(6)$, that $s_{B}^{*} \in \tilde{W}_{B}$. Hence $W_{A}\left(s_{B}^{*}\right) \cup T\left(s_{B}^{*}\right)=\emptyset$. Thus 
$s_{A}^{*}\left(s_{B}^{*}\right) \in L_{A}$-unrestricted, given (4)-, and $\left|J\left(s_{A}^{*}\left(s_{B}^{*}\right), s_{B}^{*}\right)\right|>\left|I\left(s_{A}^{*}\left(s_{B}^{*}\right), s_{B}^{*}\right)\right|$. By (1), (2), (3) and (5) we conclude that $R_{B}\left(s^{*}\right)=1$ and either $R_{A}\left(s^{*}\right)=1$ and $\sum_{j \in N_{\mathcal{B}}} v_{j}^{*}\left(s_{A}^{*}\left(s_{B}^{*}\right), s_{B}^{*}\right)>\sum_{i \in N_{\mathcal{A}}} v_{i}^{*}\left(s_{A}^{*}\left(s_{B}^{*}\right), s_{B}^{*}\right)$, or $R_{A}\left(s^{*}\right)=0$. Hence, $P_{A}\left(s^{*}\right)=0$.

(ii) $\tilde{W}_{B}=\emptyset$ and $\tilde{T} \neq \emptyset$. In such a case we know, by (6), that $s_{B}^{*} \in \tilde{T}$. Hence $W_{A}\left(s_{B}^{*}\right)=\emptyset$ and $T\left(s_{B}^{*}\right) \neq \emptyset$. Thus, given (4) we have $s_{A}^{*}\left(s_{B}^{*}\right) \in T\left(s_{B}^{*}\right)$, and $\left|I\left(s_{A}^{*}\left(s_{B}^{*}\right), s_{B}^{*}\right)\right|=\left|J\left(s_{A}^{*}\left(s_{B}^{*}\right), s_{B}^{*}\right)\right|$. By (1), (2), (3) and (5) we conclude that $R_{B}\left(s^{*}\right)=R_{A}\left(s^{*}\right)=1$ and $\sum_{i \in N_{\mathcal{A}}} v_{i}^{*}\left(s_{A}^{*}\left(s_{B}^{*}\right), s_{B}^{*}\right)=\sum_{j \in N_{\mathcal{B}}} v_{j}^{*}\left(s_{A}^{*}\left(s_{B}^{*}\right), s_{B}^{*}\right)$. Hence, $P_{A}\left(s^{*}\right)=\frac{1}{2}$.

(iii) $\tilde{W}_{B}=\tilde{T}=\emptyset$. In such a case we know, by (6), that $s_{B}^{*} \in L_{B}$ is unrestricted. Hence $W_{A}\left(s_{B}^{*}\right) \neq \emptyset$. Thus, given $(4), s_{A}^{*}\left(s_{B}^{*}\right) \in W_{A}\left(s_{B}^{*}\right)$ and $\left|I\left(s_{A}^{*}\left(s_{B}^{*}\right), s_{B}^{*}\right)\right|>\left|J\left(s_{A}^{*}\left(s_{B}^{*}\right), s_{B}^{*}\right)\right|$. By (1), (2), (3) and (5) we conclude that either $R_{B}\left(s^{*}\right)=0$ or $R_{B}\left(s^{*}\right)=R_{A}\left(s^{*}\right)=1$ and $\sum_{i \in N_{\mathcal{A}}} v_{i}^{*}\left(s_{A}^{*}\left(s_{B}^{*}\right), s_{B}^{*}\right)>$ $\sum_{j \in N_{\mathcal{B}}} v_{j}^{*}\left(s_{A}^{*}\left(s_{B}^{*}\right), s_{B}^{*}\right)$. Hence, $P_{A}\left(s^{*}\right)=1$. This completes the proof.

\section{Proof of Proposition 2}

We have defined $S^{* *}$ as the set of equilibrium strategy profiles satisfying conditions (7)-(11) defined in Section 3.2. To prove the first part of the proposition we have to show that condition (9) is equivalent to $s_{A}^{*}\left(l_{B}\right) \in \arg \max _{l_{A} \in L_{A}}\left|F\left(l_{A}\right)\right|+\left|C_{A}\left(l_{A}, l_{B}\right)\right|-\left|C_{B}\left(l_{A}, l_{B}\right)\right|$ for any $l_{B} \in L_{B}$, and condition (11) is equivalent to $s_{B}^{*} \in \arg \max _{l_{B} \in L_{B}}\left|F\left(l_{B}\right)\right|-\left|F\left(s_{A}^{*}\left(l_{B}\right)\right)\right|+\left|C_{B}\left(s_{A}^{*}\left(l_{B}\right), l_{B}\right)\right|-\left|C_{A}\left(s_{A}^{*}\left(l_{B}\right), l_{B}\right)\right|$.

Let us first prove the first equivalence. Condition (9) states that $s_{A}^{*}\left(l_{B}\right) \in \arg \max _{l_{A} \in L_{A}}\left|I\left(l_{A}, l_{B}\right)\right|-$ $\left|J\left(l_{A}, l_{B}\right)\right|$, for any $l_{B} \in L_{B}$. Note that $\left|I\left(l_{A}, l_{B}\right)\right|=\left|N_{\mathcal{A}}\right|-\left|F\left(l_{B}\right)\right|+\left|C_{A}\left(l_{A}, l_{B}\right)\right|$ and $\left|J\left(l_{A}, l_{B}\right)\right|=\left|N_{\mathcal{B}}\right|-$ $\left|F\left(l_{A}\right)\right|+\left|C_{B}\left(l_{A}, l_{B}\right)\right|$. Thus, $\left|I\left(l_{A}, l_{B}\right)\right|-\left|J\left(l_{A}, l_{B}\right)\right|=\left|F\left(l_{A}\right)\right|-\left|F\left(l_{B}\right)\right|+\left|C_{A}\left(l_{A}, l_{B}\right)\right|-\left|C_{B}\left(l_{A}, l_{B}\right)\right|+\left|N_{\mathcal{A}}\right|-$ $\left|N_{\mathcal{B}}\right|$. Therefore, the set $\arg \max _{l_{A} \in L_{A}}\left|I\left(l_{A}, l_{B}\right)\right|-\left|J\left(l_{A}, l_{B}\right)\right|$ coincides with the set $\arg \max _{l_{A} \in L_{A}}\left|F\left(l_{A}\right)\right|+$ $\left|C_{A}\left(l_{A}, l_{B}\right)\right|-\left|C_{B}\left(l_{A}, l_{B}\right)\right|$.

We now prove the second equivalence. Condition (11) states that $s_{B}^{*} \in \arg \max _{l_{B} \in L_{B}}\left|J\left(s_{A}^{*}\left(l_{B}\right), l_{B}\right)\right|-$ $\left|I\left(s_{A}^{*}\left(l_{B}\right), l_{B}\right)\right|$. Note that $\left|J\left(s_{A}^{*}\left(l_{B}\right), l_{B}\right)\right|-\left|I\left(s_{A}^{*}\left(l_{B}\right), l_{B}\right)\right|=\left|F\left(l_{B}\right)\right|-\left|F\left(s_{A}^{*}\left(l_{B}\right)\right)\right|+\left|C_{B}\left(s_{A}^{*}\left(l_{B}\right), l_{B}\right)\right|-$ $\left|C_{A}\left(s_{A}^{*}\left(l_{B}\right), l_{B}\right)\right|+\left|N_{\mathcal{B}}\right|-\left|N_{\mathcal{A}}\right|$. Therefore the set $\arg \max _{l_{B} \in L_{B}}\left|J\left(s_{A}^{*}\left(l_{B}\right), l_{B}\right)\right|-\left|I\left(s_{A}^{*}\left(l_{B}\right), l_{B}\right)\right|$ coincides with the set $\arg \max _{l_{B} \in L_{B}}\left|F\left(l_{B}\right)\right|-\left|F\left(s_{A}^{*}\left(l_{B}\right)\right)\right|+\left|C_{B}\left(s_{A}^{*}\left(l_{B}\right), l_{B}\right)\right|-\left|C_{A}\left(s_{A}^{*}\left(l_{B}\right), l_{B}\right)\right|$.

To prove the second part of the proposition, note that $s_{A}^{*}\left(l_{B}\right) \in \arg \max _{l_{A} \in L_{A}}\left|F\left(l_{A}\right)\right|+\left|C_{A}\left(l_{A}, l_{B}\right)\right|-$ $\left|C_{B}\left(l_{A}, l_{B}\right)\right|$ implies $\left|F\left(s_{A}^{*}\left(l_{B}\right)\right)\right|+\left|C_{A}\left(s_{A}^{*}\left(l_{B}\right), l_{B}\right)\right|-\left|C_{B}\left(s_{A}^{*}\left(l_{B}\right), l_{B}\right)\right| \geq\left|F\left(l_{A}\right)\right|+\left|C_{A}\left(l_{A}, l_{B}\right)\right|-\left|C_{B}\left(l_{A}, l_{B}\right)\right|$ for any $l_{A} \in L_{A}$. In particular, this must hold for $l_{A}=l_{A}^{m c}$. Then, $\left(\left|C_{A}\left(s_{A}^{*}\left(l_{B}\right), l_{B}\right)\right|-\left|C_{B}\left(s_{A}^{*}\left(l_{B}\right), l_{B}\right)\right|\right)-$ $\left(\left|C_{A}\left(l_{A}^{m c}, l_{B}\right)\right|-\left|C_{B}\left(l_{A}^{m c}, l_{B}\right)\right|\right) \geq\left|F\left(l_{A}^{m c}\right)\right|-\left|F\left(s_{A}^{*}\left(l_{B}\right)\right)\right|$. Now note that, for any $l_{A} \in L_{A}$ and $l_{B} \in L_{B}$, $\left|C_{A}\left(l_{A}, l_{B}\right)\right|=0$ implies $\left|C_{B}\left(l_{A}, l_{B}\right)\right|=0$, and $\left|C_{A}\left(l_{A}, l_{B}\right)\right| \in\{1, \ldots, k\}$ implies $\left|C_{B}\left(l_{A}, l_{B}\right)\right| \in\{1, \ldots, k\}$. Hence, $\left|C_{A}\left(l_{A}, l_{B}\right)\right| \in\{0,1, \ldots, k\}$ and $\left|C_{B}\left(l_{A}, l_{B}\right)\right| \in\{0,1, \ldots, k\}$, together with the fact that $\left|C_{A}\left(l_{A}, l_{B}\right)\right|=$ 0 implies $\left|C_{B}\left(l_{A}, l_{B}\right)\right|=0$, results in $\left|C_{A}\left(l_{A}, l_{B}\right)\right|-\left|C_{B}\left(l_{A}, l_{B}\right)\right| \in\{-k+1, \ldots, k-1\}$. Hence, for any pair of lists $l_{A}, l_{A}^{\prime},\left(\left|C_{A}\left(l_{A}, l_{B}\right)\right|-\left|C_{B}\left(l_{A}, l_{B}\right)\right|\right)-\left(\left|C_{A}\left(l_{A}^{\prime}, l_{B}\right)\right|-\left|C_{B}\left(l_{A}^{\prime}, l_{B}\right)\right|\right) \in\{-2 k+2,-2 k+3, \ldots, 2 k-2\}$. In particular, for the lists $s_{A}^{*}\left(l_{B}\right)$ and $l_{A}^{m c}$, we have $\left(\left|C_{A}\left(s_{A}^{*}\left(l_{B}\right), l_{B}\right)\right|-\left|C_{B}\left(s_{A}^{*}\left(l_{B}\right), l_{B}\right)\right|\right)-\left(\left|C_{A}\left(l_{A}^{m c}, l_{B}\right)\right|-\right.$ $\left.\left|C_{B}\left(l_{A}^{m c}, l_{B}\right)\right|\right) \leq 2 k-2$. Thus, $\left|F\left(l_{A}^{m c}\right)\right|-\left|F\left(s_{A}^{*}\left(l_{B}\right)\right)\right| \leq 2 k-2$. 
We now prove that $\left(\left|F\left(l_{B}^{m c}\right)\right|-\left|F\left(s_{B}^{*}\right)\right|\right) \leq 2 k-2$. First, note that $s_{B}^{*} \in \arg \max _{l_{B} \in L_{B}}\left|F\left(l_{B}\right)\right|-$ $\left(\left|F\left(s_{A}^{*}\left(l_{B}\right)\right)\right|+\left|C_{A}\left(s_{A}^{*}\left(l_{B}\right), l_{B}\right)\right|-\left|C_{B}\left(s_{A}^{*}\left(l_{B}\right), l_{B}\right)\right|\right)$ implies $\left|F\left(s_{B}^{*}\right)\right|-\left(\left|F\left(s_{A}^{*}\left(s_{B}^{*}\right)\right)\right|+\left|C_{A}\left(s_{A}^{*}\left(s_{B}^{*}\right), s_{B}^{*}\right)\right|-\right.$ $\left.\left|C_{B}\left(s_{A}^{*}\left(s_{B}^{*}\right), s_{B}^{*}\right)\right|\right) \geq\left|F\left(l_{B}\right)\right|-\left(\left|F\left(s_{A}^{*}\left(l_{B}\right)\right)\right|+\left|C_{A}\left(s_{A}^{*}\left(l_{B}\right), l_{B}\right)\right|-\left|C_{B}\left(s_{A}^{*}\left(l_{B}\right), l_{B}\right)\right|\right)$ for any $l_{B} \in L_{B}$.

In particular, this must hold for $l_{B}=l_{B}^{m c}$. Then, $\left(\left|F\left(s_{A}^{*}\left(l_{B}^{m c}\right)\right)\right|+\left|C_{A}\left(s_{A}^{*}\left(l_{B}^{m c}\right), l_{B}^{m c}\right)\right|-\left|C_{B}\left(s_{A}^{*}\left(l_{B}^{m c}\right), l_{B}^{m c}\right)\right|\right)-$ $\left(\left|F\left(s_{A}^{*}\left(s_{B}^{*}\right)\right)\right|+\left|C_{A}\left(s_{A}^{*}\left(s_{B}^{*}\right), s_{B}^{*}\right)\right|-\left|C_{B}\left(s_{A}^{*}\left(s_{B}^{*}\right), s_{B}^{*}\right)\right|\right) \geq\left|F\left(l_{B}^{m c}\right)\right|-\left|F\left(s_{B}^{*}\right)\right|$.

Note that, for any $l_{B} \in L_{B},\left|F\left(s_{A}^{*}\left(l_{B}\right)\right)\right|+\left|C_{A}\left(s_{A}^{*}\left(l_{B}\right), l_{B}\right)\right|-\left|C_{B}\left(s_{A}^{*}\left(l_{B}\right), l_{B}\right)\right| \geq\left|F\left(l_{A}^{m c}\right)\right|-k+1$. In particular, we have that, for $s_{B}^{*},\left|F\left(s_{A}^{*}\left(s_{B}^{*}\right)\right)\right|+\left|C_{A}\left(s_{A}^{*}\left(s_{B}^{*}\right), s_{B}^{*}\right)\right|-\left|C_{B}\left(s_{A}^{*}\left(s_{B}^{*}\right), s_{B}^{*}\right)\right| \geq\left|F\left(l_{A}^{m c}\right)\right|-k+1$. Moreover note that, for any $l_{B} \in L_{B},\left|F\left(s_{A}^{*}\left(l_{B}\right)\right)\right|+\left|C_{A}\left(s_{A}^{*}\left(l_{B}\right), l_{B}\right)\right|-\left|C_{B}\left(s_{A}^{*}\left(l_{B}\right), l_{B}\right)\right| \leq\left|F\left(l_{A}^{m c}\right)\right|+k-1$. In particular, for $l_{B}^{m c},\left|F\left(s_{A}^{*}\left(l_{B}^{m c}\right)\right)\right|+\left|C_{A}\left(s_{A}^{*}\left(l_{B}^{m c}\right), l_{B}^{m c}\right)\right|-\left|C_{B}\left(s_{A}^{*}\left(l_{B}^{m c}\right), l_{B}^{m c}\right)\right| \leq\left|F\left(l_{A}^{m c}\right)\right|+k-1$.

Hence, $\left(\left|F\left(s_{A}^{*}\left(l_{B}^{m c}\right)\right)\right|+\left|C_{A}\left(s_{A}^{*}\left(l_{B}^{m c}\right), l_{B}^{m c}\right)\right|-\left|C_{B}\left(s_{A}^{*}\left(l_{B}^{m c}\right), l_{B}^{m c}\right)\right|\right)-\left(\left|F\left(s_{A}^{*}\left(s_{B}^{*}\right)\right)\right|+\left|C_{A}\left(s_{A}^{*}\left(s_{B}^{*}\right), s_{B}^{*}\right)\right|-\right.$ $\left.\left|C_{B}\left(s_{A}^{*}\left(s_{B}^{*}\right), s_{B}^{*}\right)\right|\right) \leq 2 k-2$. Therefore, $\left|F\left(l_{B}^{m c}\right)\right|-\left|F\left(s_{B}^{*}\right)\right| \leq 2 k-2$. This completes the proof.

\section{Proof of Corollary 2}

Using a similar argument to the one used in the proof of Proposition 2, we have to show that, in the case $k=1$, condition (9) is equivalent to $s_{A}^{*}\left(l_{B}\right) \in L_{A}^{m c}$ for any $l_{B} \in L_{B}$, and condition (11) is equivalent to $s_{B}^{*} \in L_{B}^{m c}$. To this aim, note that, in the case $k=1$, we have $\left|C_{A}\left(l_{A}, l_{B}\right)\right|=\left|C_{B}\left(l_{A}, l_{B}\right)\right|$ for any $l_{A} \in L_{A}$ and $l_{B} \in L_{B}$.

$>$ From Proposition 2 we know that the set $\arg \max _{l_{A} \in L_{A}}\left|I\left(l_{A}, l_{B}\right)\right|-\left|J\left(l_{A}, l_{B}\right)\right|$ is equivalent to the set $\arg \max _{l_{A} \in L_{A}}\left|F\left(l_{A}\right)\right|+\left|C_{A}\left(l_{A}, l_{B}\right)\right|-\left|C_{B}\left(l_{A}, l_{B}\right)\right|$. Then, given that $\left|C_{A}\left(l_{A}, l_{B}\right)\right|=\left|C_{B}\left(l_{A}, l_{B}\right)\right|$ in the case $k=1$, we obtain $s_{A}^{*}\left(l_{B}\right) \in \arg \max _{l_{A} \in L_{A}}\left|F\left(l_{A}\right)\right|$ for any $l_{B} \in L_{B}$. Hence, $s_{A}^{*}\left(l_{B}\right) \in L_{A}^{m c}$ for any $l_{B} \in L_{B}$.

Analogously for candidate $B$, we have that the set $\arg \max _{l_{B} \in L_{B}}\left|J\left(s_{A}^{*}\left(l_{B}\right), l_{B}\right)\right|-\left|I\left(s_{A}^{*}\left(l_{B}\right), l_{B}\right)\right|$ is equivalent to the set $\arg \max _{l_{B} \in L_{B}}\left|F\left(l_{B}\right)\right|-\left|F\left(s_{A}^{*}\left(l_{B}\right)\right)\right|+\left|C_{B}\left(s_{A}^{*}\left(l_{B}\right), l_{B}\right)\right|-\left|C_{A}\left(s_{A}^{*}\left(l_{B}\right), l_{B}\right)\right|$, which furthermore coincides with the set $\arg \max _{l_{B} \in L_{B}}\left|F\left(l_{B}\right)\right|$, as $\left|C_{A}\left(l_{A}, l_{B}\right)\right|=\left|C_{B}\left(l_{A}, l_{B}\right)\right|$ and $s_{A}^{*}\left(l_{B}\right) \in L_{A}^{m c}$ for any $l_{B} \in L_{B}$, and then, $\left|F\left(s_{A}^{*}\left(l_{B}\right)\right)\right|$ is constant and independent of $l_{B}$. Hence, $s_{B}^{*} \in L_{B}^{m c}$. This completes the proof.

\section{Proof of Proposition 3}

Assume $k=1$. Consider first the case $\mathcal{A}=\mathcal{X}$ and $\mathcal{B}=\mathcal{Y}$. By Corollary 2, if $s^{*} \in S^{* *}$, then $s^{*}$ satisfies conditions (7), (8) and (10), $s_{A}^{*}\left(s_{B}^{*}\right) \in L_{A}^{m c}$, and $s_{B}^{*} \in L_{B}^{m c}$. Then $\left|I\left(s_{A}^{*}\left(s_{B}^{*}\right), s_{B}^{*}\right)\right|=\left|N_{\mathcal{X}}\right|-$ $\left|F\left(s_{B}^{*}\right)\right|+\left|C_{A}\left(s_{A}^{*}\left(s_{B}^{*}\right), s_{B}^{*}\right)\right|$ and $\left|J\left(s_{A}^{*}\left(s_{B}^{*}\right), s_{B}^{*}\right)\right|=\left|N_{\mathcal{Y}}\right|-\left|F\left(s_{A}^{*}\left(s_{B}^{*}\right)\right)\right|+\left|C_{B}\left(s_{A}^{*}\left(s_{B}^{*}\right), s_{B}^{*}\right)\right|$. As $k=1$ implies $\left|C_{A}\left(s_{A}^{*}\left(s_{B}^{*}\right), s_{B}^{*}\right)\right|=\left|C_{B}\left(s_{A}^{*}\left(s_{B}^{*}\right), s_{B}^{*}\right)\right|$, there are three possibilities.

(i) If $\left|N_{\mathcal{X}}\right|+\left|F\left(s_{A}^{*}\left(s_{B}^{*}\right)\right)\right|>\left|N_{\mathcal{Y}}\right|+\left|F\left(s_{B}^{*}\right)\right|$, then $\left|I\left(s_{A}^{*}\left(s_{B}^{*}\right), s_{B}^{*}\right)\right|>\left|J\left(s_{A}^{*}\left(s_{B}^{*}\right), s_{B}^{*}\right)\right|$ and, by (7), (8) and (10), $P_{A}\left(s^{*}\right)=1$.

(ii) If $\left|N_{\mathcal{X}}\right|+\left|F\left(s_{A}^{*}\left(s_{B}^{*}\right)\right)\right|=\left|N_{\mathcal{Y}}\right|+\left|F\left(s_{B}^{*}\right)\right|$, then $\left|I\left(s_{A}^{*}\left(s_{B}^{*}\right), s_{B}^{*}\right)\right|=\left|J\left(s_{A}^{*}\left(s_{B}^{*}\right), s_{B}^{*}\right)\right|$ and, by (7), (8) and (10), $P_{A}\left(s^{*}\right)=1 / 2$. 
(iii) If $\left|N_{\mathcal{X}}\right|+\left|F\left(s_{A}^{*}\left(s_{B}^{*}\right)\right)\right|<\left|N_{\mathcal{Y}}\right|+\left|F\left(s_{B}^{*}\right)\right|$, then $\left|J\left(s_{A}^{*}\left(s_{B}^{*}\right), s_{B}^{*}\right)\right|>\left|I\left(s_{A}^{*}\left(s_{B}^{*}\right), s_{B}^{*}\right)\right|$ and, by (7), (8) and (10), $P_{A}\left(s^{*}\right)=0$.

Consider now the case $\mathcal{B}=\mathcal{X}$ and $\mathcal{A}=\mathcal{Y}$. By Corollary 2, if $\hat{s}^{*} \in \hat{S}^{* *}$, then $\hat{s}^{*}$ satisfies conditions (7), (8) and (10), $\hat{s}_{A}^{*}\left(\hat{s}_{B}^{*}\right) \in \hat{L}_{A}^{m c}$, and $\hat{s}_{B}^{*} \in \hat{L}_{B}^{m c}$. Then $\left|I\left(\hat{s}_{A}^{*}\left(\hat{s}_{B}^{*}\right), \hat{s}_{B}^{*}\right)\right|=\left|N_{\mathcal{Y}}\right|-\left|F\left(\hat{s}_{B}^{*}\right)\right|+\left|C_{A}\left(\hat{s}_{A}^{*}\left(\hat{s}_{B}^{*}\right), \hat{s}_{B}^{*}\right)\right|$ and $\left|J\left(\hat{s}_{A}^{*}\left(\hat{s}_{B}^{*}\right), \hat{s}_{B}^{*}\right)\right|=\left|N_{\mathcal{X}}\right|-\left|F\left(\hat{s}_{A}^{*}\left(\hat{s}_{B}^{*}\right)\right)\right|+\left|C_{B}\left(\hat{s}_{A}^{*}\left(\hat{s}_{B}^{*}\right), s_{B}^{*}\right)\right|$. As $k=1$ implies $\left|C_{A}\left(\hat{s}_{A}^{*}\left(\hat{s}_{B}^{*}\right), \hat{s}_{B}^{*}\right)\right|=$ $\left|C_{B}\left(\hat{s}_{A}^{*}\left(\hat{s}_{B}^{*}\right), s_{B}^{*}\right)\right|$, there are three possibilities.

(i) If $\left|N_{\mathcal{X}}\right|+\left|F\left(\hat{s}_{B}^{*}\right)\right|>\left|N_{\mathcal{Y}}\right|+\left|F\left(\hat{s}_{A}^{*}\left(\hat{s}_{B}^{*}\right)\right)\right|$, then $\left|J\left(\hat{s}_{A}^{*}\left(\hat{s}_{B}^{*}\right), \hat{s}_{B}^{*}\right)\right|>\left|I\left(\hat{s}_{A}^{*}\left(l_{B}\right), \hat{s}_{B}^{*}\right)\right|$ and, by (7), (8) and $(10), P_{A}\left(\hat{s}^{*}\right)=0$.

(ii) If $\left|N_{\mathcal{X}}\right|+\left|F\left(\hat{s}_{B}^{*}\right)\right|=\left|N_{\mathcal{Y}}\right|+\left|F\left(\hat{s}_{A}^{*}\left(\hat{s}_{B}^{*}\right)\right)\right|$, then $\left|J\left(s_{A}^{*}\left(\hat{s}_{B}^{*}\right), \hat{s}_{B}^{*}\right)\right|=\left|I\left(\hat{s}_{A}^{*}\left(\hat{s}_{B}^{*}\right), \hat{s}_{B}^{*}\right)\right|$ and, by (7), (8) and $(10), P_{A}\left(\hat{s}^{*}\right)=1 / 2$.

(iii) If $\left|N_{\mathcal{X}}\right|+\left|F\left(\hat{s}_{B}^{*}\right)\right|<\left|N_{\mathcal{Y}}\right|+\left|F\left(\hat{s}_{A}^{*}\left(\hat{s}_{B}^{*}\right)\right)\right|$, then $\left|I\left(\hat{s}_{A}^{*}\left(l_{B}\right), \hat{s}_{B}^{*}\right)\right|>\left|J\left(\hat{s}_{A}^{*}\left(\hat{s}_{B}^{*}\right), \hat{s}_{B}^{*}\right)\right|$ and, by (7), (8) and $(10), P_{A}\left(\hat{s}^{*}\right)=1$.

Note that $\left|F\left(s_{A}^{*}\left(s_{B}^{*}\right)\right)\right|=\left|F\left(\hat{s}_{B}^{*}\right)\right|$, since $s_{A}^{*}\left(s_{B}^{*}\right) \in L_{A}^{m c}($ when $\mathcal{A}=\mathcal{X})$ and $\hat{s}_{B}^{*} \in \hat{L}_{B}^{m c}($ when $\mathcal{B}=\mathcal{X})$. Analogously, $\left|F\left(s_{B}^{*}\right)\right|=\mid F\left(\hat{s}_{A}^{*}\left(\hat{s}_{B}^{*}\right) \mid\right.$, since $s_{B}^{*} \in L_{B}^{m c}($ when $\mathcal{B}=\mathcal{Y})$ and $\hat{s}_{A}^{*}\left(\hat{s}_{B}^{*}\right) \in \hat{L}_{A}^{m c}$ (when $\mathcal{A}=\mathcal{Y}$ ). Hence, it is readily seen that, for any $s^{*} \in S^{* *}$ and $\hat{s}^{*} \in \hat{S}^{* *}, P_{A}\left(s^{*}\right)=1-P_{A}\left(\hat{s}^{*}\right)=P_{B}\left(\hat{s}^{*}\right)$.

To complete the proof we need to prove that this result holds for the complete sets of strong perfect equilibria, i.e. $S^{*}$ and $\hat{S}^{*}$, but this follows directly from Corollary 1.

\section{Proof of Proposition 4}

We first prove that if, for any $\left(l_{A}, l_{B}\right) \in L_{A} \times L_{B}$, either (I), (II) or (III) hold, then $S^{*} \neq \emptyset$.

Consider a strategy profile $s^{*}$ satisfying conditions (7)-(11) (cf. Section 3.2) and assume that for any $\left(l_{A}, l_{B}\right) \in L_{A} \times L_{B}$, either (I), (II) or (III) hold. We follow a backwards induction argument to show that $s^{*} \in S^{*}$.

In the third stage of the game, for any possible pair of proposed lists $\left(l_{A}, l_{B}\right) \in L_{A} \times L_{B}$, given (7), the outcome of the election is determined by the cardinalities of the sets $I\left(l_{A}, l_{B}\right)$ and $J\left(l_{A}, l_{B}\right)$. In case (I), i.e. if $\left|I\left(l_{A}, l_{B}\right)\right|=\left|J\left(l_{A}, l_{B}\right)\right|$, the outcome of the election is a tie. Clearly, no individual in the sets $I\left(l_{A}, l_{B}\right)$ and $J\left(l_{A}, l_{B}\right)$ has incentives to to be part of a deviating coalition, since by deviating to not voting such individual can only reduce the chances of her preferred alternative to get office. On the other hand, none of the remaining citizens has incentives to belong to a deviating coalition since if there is no deviation they would obtain a payoff $\frac{d}{2}$ whereas, by altering the outcome of the election, they obtain either 0 (if their preferred alternative looses) or $d-c$ (if their preferred alternative wins). Note that $\frac{d}{2}>d-c>0$. Hence, no coalition has incentives to deviate. In Case (II), i.e. if $\left|I\left(l_{A}, l_{B}\right)\right| \geq\left|N_{\mathcal{B}}\right|$, we have three possibilities: (II.a) $\left|I\left(l_{A}, l_{B}\right)\right|=\left|N_{\mathcal{B}}\right|=\left|J\left(l_{A}, l_{B}\right)\right|$; in this case, no coalition has incentives to deviate as we showed in case (I). (II.b) $\left|I\left(l_{A}, l_{B}\right)\right|=\left|N_{\mathcal{B}}\right|>\left|J\left(l_{A}, l_{B}\right)\right|$; in this case, list $l_{A}$ gets into office (no individual in $N_{\mathcal{A}}$ has incentives to be part of a deviating coalition), and the outcome of the election 
can only be altered to a tie if all the individuals in the set $N_{\mathcal{B}} \backslash J\left(l_{A}, l_{B}\right)$ deviate to vote but, in such a case their payoff would be lower $\left(\frac{d}{2}-c<0\right.$ ). (II.c) $\left|I\left(l_{A}, l_{B}\right)\right|>\left|N_{\mathcal{B}}\right|$, list $l_{A}$ gets into office (no individual in $N_{\mathcal{A}}$ has incentives to be part of a deviating coalition) and the outcome of the election cannot be altered by the individuals in the set $N_{\mathcal{B}} \backslash J\left(l_{A}, l_{B}\right)$. Hence, no coalition has incentives to deviate at this stage in case (II). Analogously, it can be shown that in case (III), $\left|J\left(l_{A}, l_{B}\right)\right| \geq\left|N_{\mathcal{A}}\right|$, no coalition has incentives to deviate at this stage.

We now turn to the second stage. Given (7), clearly for any list $l_{B} \in L_{B}$ (formed in the first stage) and any list $l_{A} \in L_{A}$ proposed by candidate $A$, no coalition of players in $l_{A}$ has incentives to deviate (in the acceptance decisions) from the behavior prescribed by (8). To see this note that $h>d>c$ implies $\frac{h+d}{2}-c>0$. Note also that, given (7)-(8), candidate $A$ does not have incentives to deviate from the behavior prescribed by (9).

Finally, we analyze the first stage. Given (7)-(9), clearly for any list $l_{B} \in L_{B}$ proposed by candidate $B$, no coalition of players in $l_{B}$ has incentives to deviate (in the acceptance decisions) from the behavior prescribed by (10), since $h>d>c$ implies $\frac{h+d}{2}-c>0$. Note also that, given (7)-(10), candidate $A$ does not have incentives to deviate from the behavior prescribed by (11).

Since by construction, the set of strategy profiles satisfying (7)-(11) is not empty, the proof of the first part of the proposition is complete.

We now prove that if there exists a pair $\left(\bar{l}_{A}, \bar{l}_{B}\right) \in L_{A} \times L_{B}$ for which neither (I), (II) nor (III) holds, then $S^{*}=\emptyset$.

Assume that such a pair $\left(\bar{l}_{A}, \bar{l}_{B}\right)$ exists, we then prove that there is no strong Nash equilibrium in the proper subgame corresponding to the third stage (voting stage) in which lists $\bar{l}_{A}$ and $\bar{l}_{B}$ have been formed. Since neither (I), (II) nor (III) holds for $\left(\bar{l}_{A}, \bar{l}_{B}\right)$ we know that $\left|N_{\mathcal{A}}\right|>\left|J\left(\bar{l}_{A}, \bar{l}_{B}\right)\right|,\left|N_{\mathcal{B}}\right|>\left|I\left(\bar{l}_{A}, \bar{l}_{B}\right)\right|$, and $\left|I\left(\bar{l}_{A}, \bar{l}_{B}\right)\right| \neq\left|J\left(\bar{l}_{A}, \bar{l}_{B}\right)\right|$. Assume for the sake of contradiction that there is a strong Nash equilibrium of the voting game $\left(v_{z}^{*}\left(\bar{l}_{A}, \bar{l}_{B}\right)\right)_{z \in N}$. There are 3 possibilities:

(i) $\sum_{i \in N_{\mathcal{A}}} v_{i}^{*}\left(\bar{l}_{A}, \bar{l}_{B}\right)>\sum_{j \in N_{\mathcal{B}}} v_{j}^{*}\left(\bar{l}_{A}, \bar{l}_{B}\right)$. Then it must be the case that no individual in the set $N_{\mathcal{A}} \backslash I\left(\bar{l}_{A}, \bar{l}_{B}\right)$ is voting, since otherwise such an individual would find it profitable to deviate to nonvoting, saving the cross-cutting cost $c$ (note that he would have incentives to deviate even if he is pivotal since $\left.c>\frac{d}{2}\right)$. Hence $\left|N_{\mathcal{B}}\right|>\left|I\left(\bar{l}_{A}, \bar{l}_{B}\right)\right| \geq \sum_{i \in N_{\mathcal{A}}} v_{i}^{*}\left(\bar{l}_{A}, \bar{l}_{B}\right)$. But, in such a case, the coalition formed by all the individuals in $\left|N_{\mathcal{B}}\right|$ who do not vote would have incentives to deviate, since $d>c$ and by deviating they win the election, a contradiction with $\left(v_{z}^{*}\left(\bar{l}_{A}, \bar{l}_{B}\right)\right)_{z \in N}$ being a strong Nash equilibrium.

(ii) $\sum_{j \in N_{\mathcal{B}}} v_{j}^{*}\left(\bar{l}_{A}, \bar{l}_{B}\right)>\sum_{i \in N_{\mathcal{A}}} v_{i}^{*}\left(\bar{l}_{A}, \bar{l}_{B}\right)$. Then it must be the case that no individual in the set $N_{\mathcal{B}} \backslash J\left(\bar{l}_{A}, \bar{l}_{B}\right)$ is voting, since otherwise such an individual would find it profitable to deviate to nonvoting, saving the cross-cutting cost $c$. Hence $\left|N_{\mathcal{A}}\right|>\left|J\left(\bar{l}_{A}, \bar{l}_{B}\right)\right| \geq \sum_{j \in N_{\mathcal{B}}} v_{j}^{*}\left(\bar{l}_{A}, \bar{l}_{B}\right)$. But, in such a case, the coalition formed by all the individuals in $\left|N_{\mathcal{A}}\right|$ who do not vote would have incentives to deviate, since $d>c$ and by deviating they win the election, a contradiction with $\left(v_{z}^{*}\left(\bar{l}_{A}, \bar{l}_{B}\right)\right)_{z \in N}$ being a strong 
Nash equilibrium.

(iii) $\sum_{i \in N_{\mathcal{A}}} v_{i}^{*}\left(\bar{l}_{A}, \bar{l}_{B}\right)=\sum_{j \in N_{\mathcal{B}}} v_{j}^{*}\left(\bar{l}_{A}, \bar{l}_{B}\right)$. Then it must be the case that no individual in the set $N \backslash\left(I\left(\bar{l}_{A}, \bar{l}_{B}\right) \cup J\left(\bar{l}_{A}, \bar{l}_{B}\right)\right)$ is voting, since otherwise such an individual would find it profitable to deviate to non-voting, saving the cross-cutting cost $c$ (recall that $\left.c>\frac{d}{2}\right)$. Hence $\left|I\left(\bar{l}_{A}, \bar{l}_{B}\right)\right| \geq \sum_{i \in N_{\mathcal{A}}} v_{i}^{*}\left(\bar{l}_{A}, \bar{l}_{B}\right)=$ $\sum_{j \in N_{\mathcal{B}}} v_{j}^{*}\left(\bar{l}_{A}, \bar{l}_{B}\right)$ and $\left|J\left(\bar{l}_{A}, \bar{l}_{B}\right)\right| \geq \sum_{i \in N_{\mathcal{A}}} v_{i}^{*}\left(\bar{l}_{A}, \bar{l}_{B}\right)=\sum_{j \in N_{\mathcal{B}}} v_{j}^{*}\left(\bar{l}_{A}, \bar{l}_{B}\right)$. Since $\left|I\left(\bar{l}_{A}, \bar{l}_{B}\right)\right| \neq\left|J\left(\bar{l}_{A}, \bar{l}_{B}\right)\right|$ we have two possibilities: First, if $\left|I\left(\bar{l}_{A}, \bar{l}_{B}\right)\right|>\left|J\left(\bar{l}_{A}, \bar{l}_{B}\right)\right|,\left|I\left(\bar{l}_{A}, \bar{l}_{B}\right)\right|>\sum_{i \in N_{\mathcal{A}}} v_{i}^{*}\left(\bar{l}_{A}, \bar{l}_{B}\right)$ and one individual in $I\left(\bar{l}_{A}, \bar{l}_{B}\right)$ who does not vote would have incentives to deviate, since by deviating his preferred alternative wins the election (recall that the voting decision does not imply any cost for any individual in the sets $I\left(\bar{l}_{A}, \bar{l}_{B}\right)$ and $\left.J\left(\bar{l}_{A}, \bar{l}_{B}\right)\right)$, a contradiction with $\left(v_{z}^{*}\left(\bar{l}_{A}, \bar{l}_{B}\right)\right)_{z \in N}$ being a strong Nash equilibrium. Analogously, if $\left|J\left(\bar{l}_{A}, \bar{l}_{B}\right)\right|>\left|I\left(\bar{l}_{A}, \bar{l}_{B}\right)\right|,\left|J\left(\bar{l}_{A}, \bar{l}_{B}\right)\right|>\sum_{j \in N_{\mathcal{B}}} v_{j}^{*}\left(\bar{l}_{A}, \bar{l}_{B}\right)$ and one individual in $J\left(\bar{l}_{A}, \bar{l}_{B}\right)$ who does not vote would have incentives to deviate, since by deviating his preferred alternative wins the election, a contradiction. This completes the proof.

\section{References}

[1] Asratian, Armen S., Tristan M.J. Denley and Roland Häggkvist (1998). Bipartite Graphs and their Applications. Cambridge University Press.

[2] Aumann, Robert J. (1959). Acceptable Points in General Cooperative n-Person Games. Contributions to the Theory of Games IV, Annals of Mathematics Study 40. A.W. Tucker and R.D. Luce (eds.). Princenton, NJ: Princeton University Press.

[3] Bernheim, B. Douglas, Bezalel Peleg and Michael D. Whinston (1987). Coalition-Proof Nash Equilibria I. Concepts. Journal of Economic Theory, 42: 1-12.

[4] Brusco, Sandro (1996). Implementing Action Profiles when Agents Collude. Journal of Economic Theory, 73: 395-424.

[5] Campbell, Angus, Phillip E. Converse, Warren E. Miller and Donald E. Stokes (1960). The American Voter. New York: Wiley.

[6] Dekel, Eddie, Matthew O. Jackson and Asher Wolinsky (2006a). Vote Buying I: General Elections. Mimeo Northwestern University.

[7] Dekel, Eddie, Matthew O. Jackson and Asher Wolinsky (2006b). Vote Buying II: General Elections. Mimeo Northwestern University.

[8] Feddersen, Timothy J. (2004). Rational Choice Theory and the Paradox of Not Voting. Journal of Economic Perspectives, 18(1): 99-112. 
[9] Fowler, James H. (2005). Turnout in a Small World. The Social Logic of Politics. Alan S. Zuckerman (ed.). Philadelphia, PA: Temple University Press.

[10] Groseclose, Tim and James M. Snyder, Jr. (1996). Buying Supermajorities. American Political Science Review, 90(2): 303-315.

[11] Herrera, Helios and César Martinelli (2006). Group Formation and Voter Participation. Mimeo ITAM-CIE.

[12] Hibbing, John R. and Elizabeth Theiss-Morse (2002). Stealth Democracy: Americans' Belief about How Government Should Work. Cambridge, UK: Cambridge University Press.

[13] Lazarsfeld, Paul F., Bernard Berelson and Hazel Gaudet (1944). The People's Choice. New York: Duell, Sloan and Pearce.

[14] Ledyard, Jonh O. (1981). The Paradox of Voting and Candidate Competition: A General Equilibrium Analysis. Essays in Contemporary Fields of Economics. G. Hoorwich and J.P. Quick (eds.). Lafayette: Purdue University Press.

[15] Ledyard, Jonh O. (1984). The Pure Theory of Large Two-Candidate Elections. Public Choice, 44(1): $7-41$.

[16] Morton, Rebecca B. (1991). Groups in Rational Turnout Models. American Journal of Political Science, 35(3): 758-776.

[17] Mutz, Diana C. (2006). Hearing the Other Side. Deliverative versus Participatory Democracy. New York, NY: Cambridge University Press.

[18] Mutz, Diana C. (2002). The Consequences of Cross-Cutting Networks for Political Participation. American Journal of Political Science, 46(4): 838-855.

[19] Palfrey, Thomas R. and Howard Rosenthal (1983). A Strategic Calculus of Voting. Public Choice, 41: $7-53$.

[20] Palfrey, Thomas R. and Howard Rosenthal (1985). Voter Participation and Strategic Uncertainty. American Political Science Review, 79: 62-78.

[21] Riker, William and Peter Ordeshook (1968). A Theory of the Calculus of Voting. American Political Science Review, 62(1): 25-42.

[22] Rubinstein, Ariel (1979). Strong Perfect Equilibrium in Supergames. International Journal of Game Theory, 9(1): 1-12. 
[23] Shachar, Ron and Barry Nalebuff (1999). Following the Leader: Theory and Evidence on Political Participation. American Economic Review, 89(3): 525-547.

[24] Simmel, Georg (1955). Conflict and the Web of Group Affiliation. Translated K.H. Wolff. New York: Free Press.

[25] Uhlaner, Carol (1989). Rational Turnout: The Neglected Role of Groups. American Journal of Political Science, 33(2): 390-422.

[26] Wolfinger, Raymond E. and Stephan J. Rosenstone (1980). Who Votes? New Haven: Yale University Press. 